\title{
Challenges and perspectives in obstructive sleep apnoea
}

Report by an ad hoc working group of the Sleep Disordered Breathing Group of the European Respiratory Society and the European Sleep Research Society

Winfried Randerath ${ }^{1}$, Claudio L. Bassetti ${ }^{2}$, Maria R. Bonsignore ${ }^{3}$, Ramon Farre ${ }^{4}$, Luigi Ferini-Strambi ${ }^{5}$, Ludger Grote ${ }^{6}$, Jan Hedner ${ }^{6}$, Malcolm Kohler ${ }^{7}$, Miguel-Angel Martinez-Garcia ${ }^{8}$, Stefan Mihaicuta ${ }^{9}$, Josep Montserrat ${ }^{10}$, Jean-Louis Pepin ${ }^{11}$, Dirk Pevernagie ${ }^{12,13}$, Fabio Pizza ${ }^{14}$, Olli Polo ${ }^{15}$, Renata Riha ${ }^{16}$, Silke Ryan ${ }^{17}$, Johan Verbraecken ${ }^{18}$ and Walter T. McNicholas ${ }^{17,19}$

@ERSpublications

Clinical and pathophysiological phenotyping and personalised diagnostic and therapeutic procedures remain challenges in obstructive sleep apnoea management http://ow.ly/OhNU30jOCr3

Cite this article as: Randerath W, Bassetti CL, Bonsignore MR, et al. Challenges and perspectives in obstructive sleep apnoea. Eur Respir J 2018; 52: 1702616 [https://doi.org/10.1183/13993003.02616-2017].

ABSTRACT Obstructive sleep apnoea (OSA) is a major challenge for physicians and healthcare systems throughout the world. The high prevalence and the impact on daily life of OSA oblige clinicians to offer effective and acceptable treatment options. However, recent evidence has raised questions about the benefits of positive airway pressure therapy in ameliorating comorbidities.

An international expert group considered the current state of knowledge based on the most relevant publications in the previous 5 years, discussed the current challenges in the field, and proposed topics for future research on epidemiology, phenotyping, underlying mechanisms, prognostic implications and optimal treatment of patients with OSA.

The group concluded that a revision to the diagnostic criteria for OSA is required to include factors that reflect different clinical and pathophysiological phenotypes and relevant comorbidities (e.g. nondipping nocturnal blood pressure). Furthermore, current severity thresholds require revision to reflect factors such as the disparity in the apnoea-hypopnoea index (AHI) between polysomnography and sleep studies that do not include sleep stage measurements, in addition to the poor correlation between AHI and daytime symptoms such as sleepiness. Management decisions should be linked to the underlying phenotype and consider outcomes beyond AHI. 
Affiliations: ${ }^{1}$ Institute of Pneumology at the University of Cologne, Bethanien Hospital, Clinic for Pneumology and Allergology, Centre of Sleep Medicine and Respiratory Care, Solingen, Germany. ${ }^{2}$ Neurology Dept, Inselspital, Bern University Hospital, Bern, Switzerland. ${ }^{3}$ DiBiMIS, University of Palermo and CNR Institute of Biomedicine and Molecular Immunology (IBIM), Palermo, Italy. ${ }^{4}$ Unit of Biophysics and Bioengineering, School of Medicine and Health Sciences, University of Barcelona-IDIBAPS, Barcelona and CIBERES, Madrid, Spain. ${ }^{5}$ Dept of Neurology OSR-Turro, Sleep Disorders Centre, Università Vita-Salute San Raffaele, Milan, Italy. ${ }^{6}$ Dept of Sleep Medicine, Respiratory Medicine and Allergology, Sahlgrenska University Hospital, Gothenburg, Sweden. ${ }^{7}$ Dept of Pneumology, University Hospital of Zurich, Zurich, Switzerland. ${ }^{8}$ Respiratory Dept, Polytechnic and University La Fe Hospital, Valencia, Spain. ${ }^{9}$ Pulmonology Dept, CardioPrevent Foundation, University of Medicine and Pharmacy Victor Babes Timisoara, Timisoara, Romania. ${ }^{10}$ Sleep Unit, Respiratory Dept, Hospital Clinic, University of Barcelona-IDIBAPS, Barcelona and CIBERES, Madrid, Spain. ${ }^{11}$ HP2 Laboratory, INSERM U1042, Grenoble Alpes University and EFCR Laboratory, Thorax and Vessels Division, Grenoble Alpes University Hospital, Grenoble, France. ${ }^{12}$ Sleep Medicine Centre, Kempenhaeghe Foundation, Heeze, The Netherlands. ${ }^{13}$ Dept of Internal Medicine, Faculty of Medicine and Health Sciences, Ghent University, Belgium. ${ }^{14}$ Dept of Biomedical and Neuromotor Sciences (DIBINEM), University of Bologna and IRCCS Institute of the Neurological Sciences, AUSL di Bologna, Bologna, Italy. ${ }^{15}$ Unesta Ltd, Tampere, Finland. ${ }^{16}$ Dept of Sleep Medicine, Royal Infirmary Edinburgh, Edinburgh, UK. ${ }^{17} \mathrm{Dept}$ of Respiratory and Sleep Medicine, St Vincent's University Hospital and School of Medicine, University College Dublin, Dublin, Ireland.

${ }^{18}$ Dept of Pulmonary Medicine and Multidisciplinary Sleep Disorders Centre, Antwerp University Hospital and University of Antwerp, Antwerp, Belgium. ${ }^{19}$ First Affiliated Hospital of Guangzhou Medical University, Guangzhou, China.

Correspondence: Walter T. McNicholas, Dept of Respiratory and Sleep Medicine, St Vincent's University Hospital, Elm Park, Dublin 4, Ireland. E-mail: walter.mcnicholasducd.ie

\section{Introduction}

Breathing disturbances during sleep present in the clinical entities of obstructive sleep apnoea (OSA), central sleep apnoea, including periodic breathing, and hypoventilation disorders [1]. These labels describe imprecisely the variety of phenotypes. Many patients suffer from different and often variable amounts of obstructive and central disturbances. The clinical entities can best be described by the definition of the pathophysiological components relevant to the individual patient, i.e. the obstruction of the upper airways, disturbances of central regulation and arousal threshold. This pathophysiological approach allows selection of optimum therapeutic options, focusing on the stabilisation of the upper airways or influencing breathing regulation and manipulating the arousal threshold. New insights in the pathophysiology of OSA and central sleep apnoea, the variety of the symptoms, and the heterogeneous treatment approaches require extensive discussion and evaluation.

Due to the high prevalence and both individual and socioeconomic healthcare issues involved, this report focuses on OSA [2]. Although already high, the prevalence of OSA is expected to further increase due to ageing of societies and the global obesity epidemic. This very high prevalence of OSA represents a challenge for diagnosis, especially as current diagnostic criteria require overnight monitoring of sleep disordered breathing, which is often limited by financial constraints. The diagnosis is further complicated by the poor association between daytime symptoms (e.g. excessive sleepiness) and the severity of OSA recorded in a sleep study [3].

Although OSA is widely recognised as an independent risk factor for cardiovascular and metabolic diseases, beneficial effects of continuous positive airway pressure (CPAP) therapy on cardiovascular outcomes in patients with established cardiovascular disease have recently been challenged [4-8]. The major early success of CPAP in the symptomatic treatment of OSA may have inhibited scientific research in many aspects of the disorder, including the complex pathophysiology and genetics, the variety of clinical presentations, and the effects and outcomes of different therapeutic options. Consequently, the diagnosis and treatment may have become oversimplified in many patients, focusing principally on the number of respiratory events during sleep and resulting in inadequate clinical characterisation. This may contribute to unclear and unexpected outcomes. Recent concepts on pathophysiology and differing clinical phenotypes of OSA provide opportunities for a better understanding and individualised therapy of the disorder [9].

At present, many patients with OSA are managed by clinicians who are not expert in sleep disorders, such as respiratory physicians who treat OSA patients as only one component of general respiratory practice, and may not have undertaken specific training in sleep disorders. This practice is facilitated by the lack of recognition of sleep medicine as a distinct speciality in many jurisdictions.

These challenges have been discussed by a group of sleep medicine specialists from the European Respiratory Society (ERS) and the European Sleep Research Society (ESRS). The aims of the process were to: 1) focus on current limitations in the diagnosis and treatment of OSA, 2) provide questions on current clinical practice, and 3) highlight future research priorities for the next decade. 


\section{Methods}

This report emanates from the deliberations of an ad hoc expert group initiated by the ERS group "Sleep and Control of Breathing" and the Alpine Sleep Summer School (sleep-summer-school.ch), a European Forum for postgraduate education and think tank activities in sleep research and medicine in the ESRS [10]. The group activities included teleconferences to coordinate actions and priorities, and literature reviews to identify the most up-to-date information on diagnosis and management, and culminated in a “Think Tank" style conference held in Baveno, Italy over 3 days in October 2016.

26 European experts were invited to participate and 19 actually attended the Baveno conference. Participants were invited based on recent activities in Task Forces of the Sleep Apnoea group of the ERS, ESRS and the Alpine Summer School, and represented a spectrum of pneumologists, neurologists and psychiatrists, in addition to basic and translational scientists. All European regions were represented to reflect a variety of healthcare systems.

In a first Delphi process performed at the outset of the project, eight major topics were defined to address the most important clinical questions and challenges: clinical phenotyping of OSA; assessment of disease severity; diagnostic algorithms/new tools; excessive daytime sleepiness (EDS) and related driving risk; OSA and neuropsychiatric disorders (NPDs); outcomes of sleep apnoea; comorbid conditions in OSA; and optimum treatment. A focus was placed on adults and on OSA. Individual topics established the task for eight working groups, each consisting of between eight and 13 participants, with individual group members contributing to several working groups.

In the next step, each expert was asked to define the most relevant papers on the respective topic during the previous 5 years. Each had to describe the most important clinical challenges and research priorities in the field of OSA for the next 5-10 years.

During the meeting in Baveno, each subgroup intensively discussed the materials gathered during the initial stages, prepared a document on their respective topics that synthesised the best current available evidence on clinical practice, and prioritised evidence gaps and research priorities to best fill these gaps. The overall output of the subgroup discussions was finally reviewed and agreed in a plenary session of all experts (figure 1).

\section{Findings and discussion}

\section{Phenotyping of OSA}

Current status and limitations of existing clinical practice

The need for an individualised approach

OSA is increasingly recognised as involving a clinical spectrum that far surpasses the classical picture of the male, obese and sleepy patient. A range of varying phenomena are now recognised that affects many aspects of the disorder:

- Pathophysiology, especially relating to pathogenesis of upper airway obstruction, loop gain and arousal threshold.

- Clinical presentation, i.e. heterogeneity of symptoms (e.g. daytime sleepiness, insomnia and mood disturbances); some patients have only minimal symptoms.

- Associated biomarkers such as inflammation, microRNA, vascular and polysomnographic parameters predicting prognosis and response to treatment.

- Comorbidities recognised to be highly associated with OSA (e.g. arterial hypertension and nondipping nocturnal blood pressure)

Current diagnostic criteria are inadequate to describe the variety of relevant subgroups, and new diagnostic tools are required to meet the increasing demand for personalised diagnosis and treatment of OSA. For personalised management, demographic factors such as age and sex must be considered, as they have a major impact in determining the clinical presentation of this disorder.

\section{Conventional diagnostic procedures}

Polysomnography (PSG) is the reference method for the diagnosis of OSA and its differential diagnosis or co-occurrence with other sleep disorders $[11,12]$. However, conventional measures of OSA severity (e.g. the apnoea-hypopnoea index (AHI)), do not correlate well with the severity of clinical symptoms. This observation evokes some concerns regarding the assessment of OSA based on PSG:

- PSG measures of severity such as AHI may need recalibration by which new cut-off limits may be established.

- Other markers than AHI could have better predictive power for disease severity. 


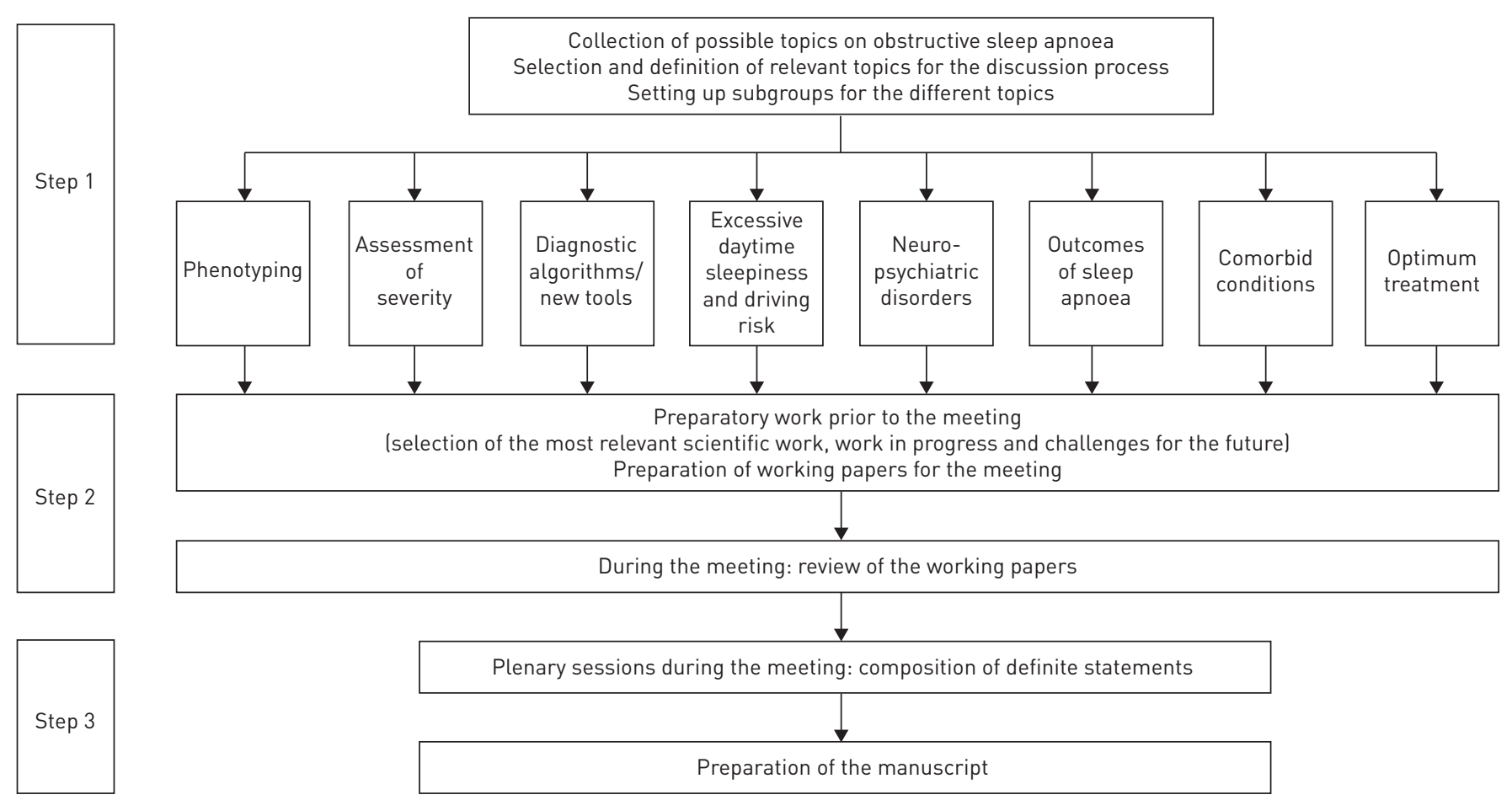

FIGURE 1 Delphi process performed at the outset of the project to select topics addressing the most important clinical questions and challenges in obstructive sleep apnoea.

- PSG may be inadequate, as it may fail to demonstrate individual susceptibility for systemic effects of OSA.

- A new conceptual framework for assessing disease severity of OSA may be elaborated. OSA may be conceived as a model of chronic recurrent strain in which the outcomes are defined by the presence of pathological stressors, on the one hand, and the tolerance to these effects, on the other hand (figure 2).

Polygraphy, while having the advantage over PSG of being more suitable for ambulatory studies, lacks the recording of neurophysiological signals. As such, polygraphy is not specifically a sleep study, as sleep stages and electroencephalogram (EEG) arousals cannot be documented. Assessment of AHI is intrinsically imprecise [13]. Coexisting sleep disorders such as insomnia, periodic limb movements and parasomnias go undetected. In conclusion, polygraphy is a relatively crude diagnostic method and unsuited for the differentiation of clinical OSA subtypes in a significant number of cases.

\section{Pathophysiological phenotypes}

For many years the pathogenesis of OSA was deemed to be the result of anatomical factors associated with an imbalance of forces acting on the upper airway, whereby a narrowed oropharyngeal airway results in increased closing pressures that overcome the ability of the upper airway dilating muscles to maintain a patent airway. Anatomical factors that contribute to upper airway narrowing include retrognathia and adenotonsillar hypertrophy, but in many patients there is a nonspecific narrowing that can be clinically evaluated by the Mallampati score. The net result of this disparity is an increased critical closing pressure, which is representative of the anatomical status. Recently, the relevance of physiological, nonanatomical factors has been investigated and several additional factors identified [14]:

- Inadequate responsiveness of the genioglossus dilating muscle.

- Decreased arousal threshold.

- Instability of the respiratory control system.

These additional nonanatomical factors have clinical relevance as they may be amenable to specific treatment approaches [9, 15]. Different morphological changes (e.g. enlargement of upper airway soft tissue structure, craniofacial structures and the variable interaction between these structures) may define different anatomical phenotypes. Three-dimensional imaging of the upper airway anatomy may help in selecting specific treatment modalities [16]. Novel techniques have recently been reported that facilitate the evaluation of these nonanatomical factors (e.g. loop gain in the ambulatory setting). It is currently 


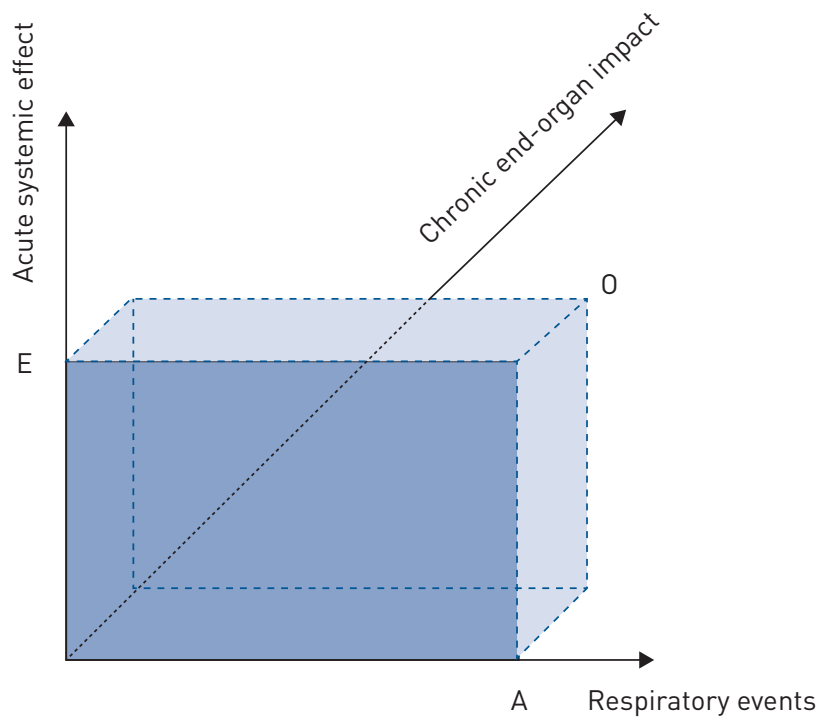

FIGURE 2 Graphic representation of a three-dimensional model of obstructive sleep apnoea (OSA) disease severity. The first dimension ( $x$-axis) represents the amount of respiratory events $(A)$ in an overnight sleep period. Usually this number is expressed in relation to total sleep time le.g. the apnoea-hypopnoea index (AHI)), which, as such, is a measure of the "density" of the respiratory events in the overnight sleep period. The second dimension ( $y$-axis) represents an acute systemic effect (E) induced by respiratory events (e.g. a certain degree of oxygen desaturation $(x \%))$. Respiratory pressure swings, arousals, changes in blood pressure, recurrent sympathetic activation and other phenomena are also part of the constellation of acute bodily effects caused by respiratory events. The shaded area AxE represents a combination of both dimensions. Combined measures, such as $x \%$ oxygen desaturation index, contain information of both frequency and amplitude of the specific effect, and may indicate the "intensity" of OSA. Such combined measures may better predict disease severity than the unidimensional $\mathrm{AHI}$. The third dimension (z-axis) represents a chronic end-organ impact $(0)$ of OSA (e.g. chronic arterial hypertension, vascular damage, insulin resistance, etc.). This impact is variable among OSA patients, even when OSA is stratified for density or intensity. The enclosed volume $A \times E \times 0$ represents the relation between intensity and the observed end-organ impact. This relationship may reflect individual susceptibility, and may comprise a spectrum between low intensity/high impact and high intensity/ low impact. The dashed aspect of the boundaries indicates that the end-organ impact of OSA is as-yet difficult to assess, because of the confounding influence of other disease processes. Specific biomarkers of end-organ damage in OSA need to be developed and validated in this research area.

unknown whether these findings are reproducible over time and between different research groups. Therefore, more studies by independent investigators are needed.

\section{Clinical phenotypes}

The clinical manifestations of OSA are very diverse, and reflect anthropometric features, comorbid conditions and environmental factors, including smoking habits, and a sedentary lifestyle. Clustering of symptoms and comorbidities allows discrimination between clinical phenotypes that include different characteristics:

- Paucity of symptoms versus EDS.

- Complaints of sleep disturbance versus undisturbed sleep.

- Presence or absence of arterial hypertension, cardiometabolic complications or severe obesity.

To date, several cluster analyses have been performed, which show mixed results [17-22]. The generalisability of available studies on clinical phenotypes is limited due to methodological differences, and prospective studies will be required to assess 1) the optimal cluster techniques and 2) the validity of cluster analysis in terms of clinical outcomes.

While EDS is a key symptom in many OSA patients, a working definition of this phenomenon is still lacking in clinical practice and clinical tools for its assessment are insufficient. More specifically, a question regarding fitness to drive should be implemented in the assessment.

\section{Assessing target organ consequences}

The link between the frequency of OSA (as measured by AHI) and clinical manifestations of OSA has proved elusive. Patients with a high AHI may be low on symptom scales and vice versa [3]. This diversity may be explained by differences in individual susceptibility to the systemic effects of OSA (e.g. intermittent hypoxia, intrathoracic pressure swings, variations in sympathetic tone and haemodynamic instability). Substances from 
molecular pathways that are triggered by these mechanisms may be identified that could serve as biomarkers reflecting the end-organ strain or damage inflicted by OSA. Biomarkers, either single or clustered, could serve as surrogate end-points for disease severity and susceptibility, as well as individual responsiveness to treatment [23]. While data on the presence of certain biomarkers in OSA have been published and the effect of treatment has been explored, research into this area is still at an early stage [24-28].

Priorities for future research

Although recent investigations have demonstrated promising results, phenotyping of OSA is not yet ready for daily practice. Remaining problems include:

- Re-definition of the optimum role of PSG.

- Clinical importance of pathophysiological traits in OSA.

- Confirmation of the current findings regarding cluster analysis in prospective cohorts.

- Clarification of the purported end-organ susceptibility for systemic effects of OSA.

- Translation of the OSA subtypes into personalised medicine.

\section{Assessment of severity of adult OSA \\ Current status and limitations of existing clinical practice}

The clinical definition of OSA based on the combination of AHI and daytime symptoms [29], particularly EDS, is compromised by the high prevalence of elevated AHI in the general population and by the poor correlation of EDS with AHI [30,31]. AHI as a measure of OSA is limited by the inclusion of arousal in the definition, making PSG the optimum modality for assessment, which does not reflect the trend towards ambulatory monitoring in clinical practice, although some ambulatory monitoring systems do include monitoring of the EEG [32]. The oxygen desaturation index (ODI) may be a stronger and more reliable predictor of adverse cardiovascular outcomes than AHI [33,34], and is easier to measure, although current guidelines continue to refer to AHI as the primary measure of OSA severity. A consensus on how to assess EDS (and other daytime consequences) in patients with OSA is lacking.

Current severity grading of OSA based on AHI [29] is influenced by the inclusion of sleep staging in the test [13] and studies that do not include sleep staging (e.g. cardiorespiratory polygraphy) give a lower AHI compared with the calculation based on PSG where periods of wakefulness during the sleep study are excluded in the calculation of AHI [13]. This difference is particularly important in the assessment of patients with mild/moderate OSA [35]. However, technological developments in electrode placement and automated analysis have facilitated the use of PSG in ambulatory recordings [36]. Multiple night studies may provide a more accurate assessment than a single night [37], which may be more feasible with the development of newer technologies utilising minimal contact devices suitable for home studies that do not include sleep staging. Technical differences (e.g. thermistor and nasal prongs) influence the assessment of respiratory disturbances and differences in hypopnoea definition may also influence the assessment of severity. The choice of $3 \%$ or $4 \%$ oxygen desaturation with or without arousal in the definition of hypopnoea $[38,39]$ has recently been demonstrated in a Spanish community-based study to substantially influence OSA prevalence and severity classification, and also affect the association with cardiovascular outcomes [40]. Oxygen desaturation is more pronounced during apnoea compared with hypopnoea and is also more pronounced with longer event duration. Severity of oxygen desaturation events differs between hypopnoea and obstructive apnoea events, and is modulated by their duration in OSA [41, 42].

The Epworth Sleepiness Scale (ESS) is the most widely used clinical tool to evaluate subjective sleepiness, but correlates poorly with AHI [43] and with objective tests of EDS, and is also open to reporting bias. Questionnaires such as the ESS may provide more accurate results with additional input from a partner. Focused questions by the clinician on core features of sleepiness (e.g. the presence of sleepiness when alone and inactive, when mentally or physically active in company, and when performing high-risk activities such as driving) may be more reliable than the ESS. Daytime symptoms in OSA are influenced by age, sex and the presence of other comorbidities [2], particularly depression and insomnia, and other symptoms such as fatigue and tiredness may be equally important in certain groups.

Anthropometric and other objective variables such as age, sex, body mass index, neck circumference and comorbidities may be more reliable than subjective variables such as snoring and EDS in predicting OSA [44]. The identification of clinically significant OSA may be improved by the inclusion of relevant comorbidities, particularly systemic hypertension, and the loss of nocturnal blood pressure dipping may add to the clinical significance of OSA (figure 3) [45].

Priorities for future research

- Definition of most appropriate diagnostic criteria to evaluate a suspected patient with OSA. 


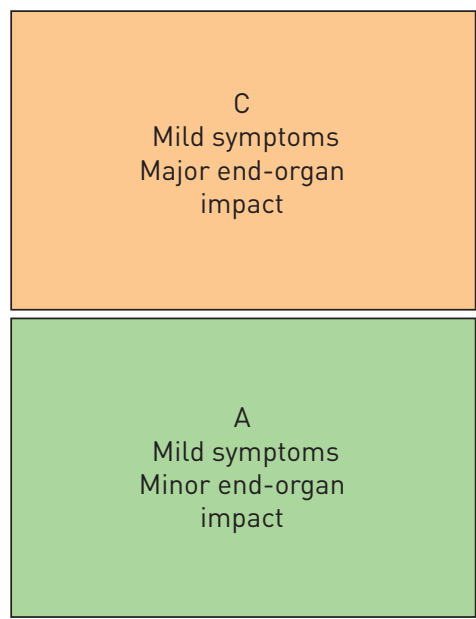

ESS $<9$

Dozing episodes-

No hypersomnia

Normal vigilance test Insomnia-

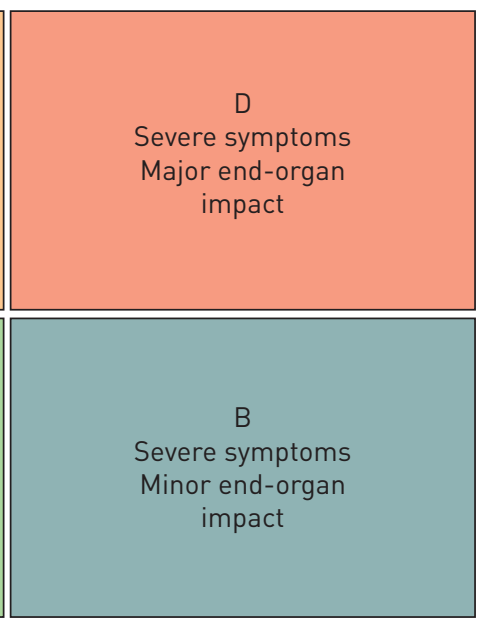

Recurrent/ poorly controlled

Not detectable/ well controlled
ESS $\geqslant 9$

Dozing episodes+ Hypersomnia

Pathological vigilance test Insomnia+

Symptoms

FIGURE 3 Proposal of a multicomponent grading system for obstructive sleep apnoea (OSA) severity. ESS: Epworth Sleepiness Scale. Prerequisite for the following grading system is the evidence of obstructive sleep-related breathing disturbances (apnoea-hypopnoea index $\geqslant 15$ events $\cdot h^{-1}$ ). The proposal combines the symptomatology based on the patient's history, the ESS, episodes of dozing off during daytime and results of objective vigilance tests. In addition, it includes the impact of OSA on the cardiovascular system and metabolism and any accompanying comorbidities. The patient is considered to suffer from mild symptoms if all conditions (ESS $<9$, no dozing episodes, no self-assessed hypersomnia, normal vigilance test) are fulfilled, whereas symptoms are considered severe if any of these parameters are positive. Patients with mild symptoms are classified as group $\mathrm{A}$ or $\mathrm{C}$ depending on the presence of comorbidities or end-organ damage. If there is no or well-controlled arterial hypertension, no or non-recurrent atrial fibrillation, no heart failure, no diabetes, or no history of a stroke, the disease is classified as minor end-organ impact leading to group $A$ or $\mathrm{B}_{\text {; }}$ if any of these factors are fulfilled, the disease is classified as major end-organ impact leading to group C or D.

- Revision of OSA severity grading to supersede the original "Chicago criteria" and subsequent updates $[29,38]$.

- Definition of the relative strengths of AHI and ODI in assessing OSA severity, particularly relating to comorbidities.

- Revision of the grading of OSA severity to reflect the presence or absence of sleep assessment/staging in the diagnostic test.

- Definition of the role of comorbidities (e.g. nondipping blood pressure) and of biomarkers in assessing the clinical significance of OSA.

- Definition of surrogates of neurovegetative activity and cardiovascular variability to detect the harmful effects of OSA.

\section{Diagnostic algorithms/new tools}

Current status and limitations of existing clinical practice

At present, an elevated AHI remains the principal objectively measured variable in diagnosis, although patients are referred for different reasons relating to daytime and/or night-time symptoms such as snoring and/or EDS, existing cardiometabolic risk, and driving or work safety issues. According to the pre-test probability of OSA, different diagnostic pathways can be applied, which may vary between centres. Currently available screening questionnaires may assist in patient pre-selection for sleep studies, but should not replace the individual patient clinical assessment [46].

There is unanimous consensus among the expert group that AHI alone is insufficient to answer three key questions:

- Who suffers from a clinically significant OSA?

- Who should be treated?

- What is the optimal therapy? 
Challenges of existing technologies and patient care

Respiratory variables other than AHI, such as flow limitation or other nonapnoeic events, might allow a more accurate assessment of OSA and new technologies may simplify the assessment of flow limitation without measuring AHI [47]. ODI and other variables of oxygen desaturation, such as cumulative sleep time with oxygen saturation below $90 \%$, minimal oxygen saturation and mean oxygen desaturation, also provide important clinical information to better address disease severity and risk of comorbidity in patients with comparable AHI [48].

New measurements and technologies may better assess the various pathophysiological mechanisms underlying OSA (e.g. loop gain, arousal threshold and anatomical factors). Reliable techniques to monitor sleep structure and arousals outside the conventional EEG (e.g. arterial tonometry) require further evaluation and development, in addition to assessment of autonomic state and cardiovascular events associated with OSA events (e.g. ECG algorithms, heart rate variability, arterial tonometry and capnography), which are currently omitted from conventional PSG [49]. Further development of existing signals such as cordless portable acoustic devices will allow enhanced diagnostic potential $[50,51]$. There is a need to identify and validate physiological signals during wakefulness or sleep that may be useful in predicting cardiovascular risk. Tools to evaluate sleepiness, including objective vigilance and performance tests, can also be considered in populations such as individual clinic patient assessment or targeted populations (e.g. those at potential driving risk). Signals in sleep studies that may better relate to EDS are required, and could include measures of sleep fragmentation, micro sleeps and/or arousal, but these require further development and validation.

Treatment responses are difficult to predict from existing diagnostic variables (e.g. AHI) and current diagnostic algorithms, which are typically one dimensional, are not adapted to the heterogeneous patient populations that include symptomatic patients, screening high-risk groups with cardiometabolic disease or patients with neuropsychiatric diseases. The potential role of new technologies (e.g. smartphone-based applications and telemedicine) is not yet sufficiently elaborated and healthcare systems often do not integrate objective information provided by the patient from self-made home recordings such as from smartphone applications.

New technologies

The following promising modalities for improved sleep diagnostics are identified, most of which require further evaluation and validation:

- Peripheral arterial tonometry to assess OSA and other sleep disorders.

- Overnight pulse wave analysis to assess autonomic and cardiovascular function/risk.

- Indwelling ear sensor to monitor the EEG.

- Capnometry for new areas in assessing sleep function and autonomic dysfunction.

- Alternative OSA screening technology, including noncontact sensor technology, acoustic breath analysis and smartphone-based applications.

- Smartphone-based diagnostic applications for sleep disorders and OSA.

- Assessment of OSA based on ambulatory Holter ECG devices or thoracic impedance from implantable devices.

- New algorithms for snoring detection, quantification and characterisation.

- Novel algorithms for the quantification of loop gain and arousal threshold.

- Biomarkers (e.g. exhaled breath analysis or RNA microarray).

Priorities for future research

- Relevant role of new technologies on existing diagnostic algorithms for OSA and targets of therapy.

- Evaluation of different diagnostic algorithms in different populations (e.g. patients with cardiovascular or other diseases, or with predominant sleepiness).

- Development of a composite OSA score, including AHI, symptoms and comorbidities/complications, to customise treatment and predict treatment responses.

- Evaluation of physiological markers of autonomic and cardiovascular function in the identification of increased cardiometabolic risk.

- Evaluation of OSA-related biomarkers in the identification of clinically relevant OSA.

- Evaluation of new digital technology (e.g. smartphone-based diagnostic technologies and treatment surveillance by telemedicine) to change sleep medicine procedures and models of care.

\section{OSA, excessive daytime sleepiness and driving}

Current status and limitations of existing clinical practice

Sleepiness is a physiological subjective sensation linked to the inner sleep need, and is influenced by circadian and homeostatic factors, external conditions, and individual features. EDS is a pathological 
disabling condition with important influences relating to sex, age and comorbidities such as depression and other disorders commonly associated with fatigue [52, 53]. EDS is associated with a subjective feeling and may also be associated with impaired psychomotor performance that may compromise driving safety.

OSA frequently causes EDS, the latter being possibly influenced by other comorbidities and masked sleep disorders, and the strength of the association is clearly related both to the operational definition of EDS itself and to the studied population [22, 54]. Most of the available literature applied the ESS, a subjective trait sleepiness assessment questionnaire on individual dozing-off attitudes in active and passive conditions. Despite its simple and wide application, the ESS is limited by a poor correlation with OSA presence and severity at the individual level, while other subjective tools addressing vigilance recently gave more promising results $[55,56]$.

Subjective EDS assessment should include partner-assisted reports, and an extensive clinical interview on habitual sleep patterns and core symptoms frequency in passive and active situations at different circadian times $[57,58]$. Objective tools include in-laboratory approaches (maintenance of wakefulness test (MWT) and multiple sleep latency test (MSLT)) validated for specific diagnostic (MSLT to characterise suspected hypersomnias of central origin) or safety-related (MWT to address individual ability to resist sleep in monotonous conditions) purposes and several nonvalidated psychomotor tests including simulated driving $[59,60]$. EDS is indeed an intrinsic marker of OSA severity, and might be a useful marker of cardiovascular and mortality outcomes [61,62]. EDS is rapidly resolved by appropriate OSA treatment, but sometimes persists. In such cases depression, inappropriate lifestyles or other undiagnosed sleep disorders should be carefully explored [52].

Pro-inflammatory cytokines (interleukin-6) appear to promote sleepiness, while cortisol promotes vigilance. It has been demonstrated that objective EDS (as measured by the MSLT), but not subjective EDS, is associated with significantly elevated 24-h interleukin-6 levels and significantly decreased daytime cortisol levels in patients with OSA [63]. Data on orexin are inconsistent in OSA, while it is an important biomarker in narcolepsy-cataplexy [64]. EDS has been recognised as an important cause in the multifactorial car accident risk in OSA patients [65]. Patients with OSA show an average 2.5-fold risk for car accidents compared with healthy controls [66]. An ESRS survey in 19 countries reported that $17 \%$ of respondents had fallen asleep at the wheel in the previous 2 years [67]. Younger age, male sex, driving at least $20000 \mathrm{~km}$ per year, higher EDS and high risk for OSA as assessed by questionnaires predicted EDS at the wheel. As shown in the European Sleep Apnea Database (ESADA) cohort, driving accident risk increased with OSA severity [68].

OSA treatment significantly reduced the risk, although whether treated OSA patients have a car accident risk comparable to the general population is strongly suggested but still under debate [69]. Prediction of individual car accident risk in OSA is the challenge. There is established evidence linking self-reported near-miss accidents with increased car accident risk $[65,70]$, while other factors such as sleepiness at the wheel and driving habits need further verification and should be addressed. In the experimental settings (on the road and simulated), driving performance was best correlated with objective vigilance (MWT) and was improved by effective OSA treatment [69], as well as by vigilance-promoting medications [71, 72]. Given the key role of individual behaviour while driving, OSA patients should understand their personal responsibility if driving while feeling sleepy, in order to avoid dangerous behaviours [59]. Recently introduced regulations by the European Union (EU) relating to driving among patients with OSA recognise that disease severity measured by both $\mathrm{AHI}$ and sleepiness are relevant to the question of driving licence restriction in such patients [73]. CPAP treatment significantly reduces EDS and driving risk in OSA patients. The EU Directive mandates objective assessment of compliance to CPAP in OSA patients. The reassessment of fitness to drive and compliance to treatment is mandatory at 3-year intervals in noncommercial drivers and every year in commercial drivers $[32,73]$. More strict rules are allowed in the individual EU member states.

Priorities for future research

Short-term priorities

- Definition and evaluation of subjective tools to reliably assess EDS in the general population.

- Identification of subjective and objective tools for reliable EDS and fitness to drive assessment in OSA patients.

- Identification of educational strategies to adequately improve OSA patient awareness on sleepiness-related accident risk.

- Identification of appropriate driving risk prediction in OSA. 
Long-term priorities

- Evaluation of patient-tailored interventions for adequate risk management.

- Assessment of functional biomarkers for EDS assessment in OSA patients.

- Identification of causes and evaluation of adequate treatments of residual EDS in well-treated OSA patients.

- Integration of OSA and EDS comprehension into transportation and industrial development standards.

\section{OSA and neuropsychiatric disorders}

Current status and limitations of existing clinical practice

There is growing evidence for an increased frequency of OSA in a variety of NPDs, including stroke, neurodegenerative/muscular disorders, major depression and post-traumatic stress disorder [10, 74-77]. Age, sex and criteria of sleep recording/scoring partially explain some of the discrepancy in the literature. However, the awareness of NPDs in OSA, and of OSA in NPDs, is not very high among specialists treating these patients $[10,78]$.

Several studies suggest that OSA may not only be frequent, but also represents an (independent) risk factor for the subsequent development of NPDs such as stroke, dementia and depression [76, 78-82].

Concerning the relationship between OSA and neurodegenerative diseases, recent studies suggest that hypoxic events can be either neuroprotective or neurotoxic, depending on several factors, including time, severity and duration of hypoxia [83, 84]. Chronic intermittent hypoxia has been associated with increased neurodegeneration related to elevated oxidative stress, as well as with neuroinflammation in animal models of sleep apnoea. Elevated oxidative stress and inflammation are hallmarks of neurodegenerative diseases $[85,86]$. In humans, intermittent hypoxia may play a role in Alzheimer's pathology. It has been associated with a cerebral increase of phosphorylated \total tau and amyloid $\beta 1-42$ concentrations in cognitively healthy adults $[87,88]$.

OSA was shown to be associated not only with EDS and fatigue, but also with an impairment of neurocognitive functions (e.g. memory and attention), psychiatric disturbances and changes of cerebral structures $[10,83,89,90]$.

The origin of such complex relationships between OSA, NPDs, cognitive and psychiatric disturbances is unclear, and may include such factors as sleep fragmentation, recurrent hypoxias, shared signalling pathways, cerebral hypoperfusion/microvascular changes, comorbidities (obesity, insomnia and EDS), medications and psychoreactive factors [91].

The clinical relevance of the link between untreated OSA and NPDs stems from the observation of a negative effect of OSA on the evolution of NPDs such as stroke, epilepsy, dementia and depression [78, 92].

Data on the effect of OSA treatment (e.g. CPAP) on NPDs is limited and contradictory [78, 93-97]. The difficulty in consistently showing an effect of OSA treatment on NPDs and their progression may be related to a variety of factors, including their chronicity, patient selection based only on AHI, heterogeneity of OSA and different outcome tools.

\section{Priorities for future research}

- Identification of neurophysiological and molecular mechanisms underlying the bidirectional link between OSA and NPDs.

- Identification and evaluation of screening tools for NPDs in the management of patients with OSA.

- Identification and evaluation of screening tools for OSA in patients with NPDs.

- Evaluation of short- or long-term benefits in treating OSA in patients with NPDs.

- Identification of causes and possible treatment options for patients with persisting EDS and NPDs despite efficient OSA therapy.

\section{Comorbid conditions in OSA}

Current status and limitations of existing clinical practice

OSA is highly prevalent in cardiovascular and metabolic diseases

Intermittent hypoxia, the hallmark of OSA, causes oxidative stress, and consequently promotes inflammation, sympathetic hyperactivity and endothelial dysfunction, which in turn lead to cardiometabolic comorbidities [4]. As demonstrated by epidemiological data in the general population and clinical cohort studies, OSA prevalence is up to $50 \%$ in arterial hypertension, refractory arrhythmias, stroke, coronary heart disease and cardiac failure $[2,85,98,99]$. There is an independent association of 
OSA with components of the metabolic syndrome [100], particularly visceral obesity, insulin resistance and abnormal lipid metabolism $[5,26]$. OSA is independently associated with alterations in glucose metabolism and increased risk of developing type 2 diabetes, with more than $50 \%$ of patients with type 2 diabetes exhibiting OSA [101, 102]. Nonalcoholic fatty liver disease is a highly prevalent condition increasing in parallel with the epidemic of obesity and type 2 diabetes. There is now evidence both in adults and children [103-105] for a link between nonalcoholic fatty liver disease and the presence of OSA, with a dose-response relationship between the severity of nocturnal hypoxia and liver injury [106, 107]. Comorbidities are of major importance, because they have a significant impact on healthcare use and mortality in patients with OSA [108, 109].

OSA is commonly associated with inflammatory lung disorders

In recent years, asthma and idiopathic pulmonary fibrosis (IPF) have been identified as significant comorbidities of OSA. The prevalence of OSA is higher in asthmatic patients than in the general population, and OSA has been associated with daytime sleepiness, poor asthma control and reduced quality of life [110-114]. Experimental studies indicate that chronic intermittent hypoxia may promote allergen-induced airway inflammation and airflow limitation [115], suggesting a detrimental effect of coexisting asthma and OSA. Both diseases considerably increase the economic burden of care [116].

Several studies on small patient samples have highlighted that mild/moderate OSA frequently occurs in IPF patients [117] and may contribute to worsen prognosis, especially when associated with significant nocturnal hypoxaemia [118]. Chronic intermittent hypoxia has been reported to exacerbate bleomycin-induced lung fibrosis in rats [119]. However, it has been hypothesised that OSA may introduce subclinical alveolar injury due to mechanical alveolar stretch, supported by increased plasma KL-6 [120].

Oxygen desaturation in patients with OSA and IPF was found to be more severe during sleep than during exercise [121]. To better understand whether OSA treatment may positively affect symptoms and prognosis of asthma or of IPF, randomised controlled studies in well-characterised samples are still needed.

The combination of COPD and OSA (the so-called "overlap syndrome") $[122,123]$ is favoured by upper airway inflammation consecutive to smoking, rostral fluid shift, upper airway muscle weakness and inhaled corticosteroids, while the predominant COPD emphysema phenotype protects against OSA. Overlap syndrome is characterised by more pronounced nocturnal hypoxaemia, oxidative stress and systemic inflammation, such that COPD patients with comorbid OSA are at increased risk for repeated exacerbations and death. Overlap also represents a major burden in survivors from acute hypercapnic respiratory failure, with a higher risk of death and early readmission [124]. CPAP treatment may improve the prognosis of patients with overlap syndrome and decrease exacerbations [125].

Epidemiological studies and animal trials suggest a link between OSA and cancer mortality and incidence [126]. A large cohort of 4910 subjects identified a significant association between overnight hypoxia and all-type cancer incidence [127]. However, a recent meta-analysis did not find a significant relationship, and studies are limited particularly for the inadequate control for obesity and the limited differentiation between different types of cancers [128].

\section{Association of OSA and comorbidities may magnify the cardiometabolic risk aggravating morbidity and mortality}

Optimal blood pressure control is more difficult to achieve in hypertensive OSA patients $[129,130]$ and there is a high prevalence of drug-resistant hypertension in the OSA population. Among the deleterious consequences of OSA, the most alarming are arrhythmias and sudden cardiac death. Rates of atrial fibrillation with poor response to medications or exhibiting recurrence of atrial fibrillation after electrical cardioversion or ablation are higher in patients with OSA [131]. Poorly controlled type 2 diabetes is also more frequent in the presence of severe OSA [132]. The number of hospitalisations and the mortality rate increase in cardiac failure patients with associated OSA [133]. In stroke patients, sleep apnoea has a negative impact on long-term outcome [78]. The comorbid condition of COPD and OSA is characterised by heightened inflammation and increased cardiovascular risk, reflecting the synergistic deleterious impact of both diseases [134].

\section{Priorities for future research}

- Evaluation of the relevance of obesity and OSA on cancer, and the specific relevance of intermittent hypoxia and sleep fragmentation in humans based on large epidemiological studies coupled with pre-clinical models.

- Evaluation of an independent cardiometabolic risk factor in OSA. 
- Evaluation of the risk associated with OSA compared with sedentary behaviour, obesity, smoking and other factors.

- Evaluation of the inclusion of OSA in integrated cardiometabolic risk reduction management.

- Evaluation of the epidemiology of the comorbid condition of COPD and OSA, considering stratification for OSA and COPD severity.

- Evaluation of a specific COPD-OSA overlap syndrome based on pathophysiological or prognostic factors.

- Relevance of OSA/intermittent hypoxia on outcomes and relative biomarkers in COPD patients.

- Definition of subgroups benefitting from CPAP treatment, especially resistant hypertension, atrial fibrillation, poorly controlled type 2 diabetes, overlap syndrome (COPD-OSA), bronchial asthma and IPF.

- Evaluation of the effect of CPAP on cardiometabolic risk in primary prevention.

\section{Treatment and outcomes}

Current status and limitations of existing clinical practice

OSA treatment has limited impact in reducing cardiometabolic risk

CPAP and other therapies for OSA have been viewed as benefitting sleepiness and other aspects relating to quality of life, in addition to reducing risk of comorbidity and mortality [135-138]. As OSA is clearly associated with metabolic and cardiovascular conditions, an effective treatment of OSA may then represent an important target for improving cardiometabolic risk. The impact of CPAP, the first-line therapy of OSA, on cardiovascular or metabolic consequences is limited and still under debate [42, 139]. The impact of CPAP or oral appliance on blood pressure is small $(-2 \mathrm{mmHg}$ for mean $24 \mathrm{~h}$ blood pressure) [137], being clinically relevant only in the resistant hypertension population [138]. Reported benefits are best in CPAP-compliant patients and especially compliance sufficient to cover rapid eye movement periods at the end of the night $[140,141]$. Whereas earlier reports indicated that fixed CPAP was superior to autoadjusting CPAP in reducing blood pressure, a recent report indicates that fixed CPAP has equal effects to autoadjusting CPAP in lowering blood pressure levels [142-144]. Mandibular advancement device (MAD) therapy has also been reported to benefit blood pressure on OSA patients [145].

CPAP does not appear to improve lipid profile or metabolic syndrome in unselected OSA populations [26]. Regarding glucose control, improvements were reported only in subgroups with suboptimally controlled type 2 diabetes [140] and prolonged nocturnal CPAP use allowing to cover REM sleep periods at the end of the night [141]. It is not realistic to expect a clinically relevant decrease in cardiometabolic mortality in secondary prevention with sole CPAP therapy as suggested by the SAVE study [6]. It did not report any benefit from CPAP therapy in reducing the incidence of future cardiovascular and cerebrovascular events in OSA patients with established cardiovascular and cerebrovascular disease, but was compromised by poor treatment compliance and low EDS, while other reports indicate that CPAP improves cardiovascular outcomes in patients with established cardiovascular disease who adequately comply with therapy $[6,22]$. The recent data demonstrating lack of efficacy of CPAP in the secondary prevention of cardiovascular disease do not exclude the possibility of benefit in the primary prevention of cardiovascular disease, although very large patient numbers may be required to evaluate this aspect. In the face of limited resources for future trials, carefully designed registries with "big data", based on real-life observations (e.g. ESADA), could be a more pragmatic approach. In trials, to overcome poor adherence leading to poor outcome, a run-in phase to optimise adherence could be justified. The negative findings of the SAVE trial may also justify prospective randomised controlled studies of more symptomatic patients with moderate/severe OSA, where treatment compliance may be expected to be higher.

A major issue is that some patients show huge CPAP treatment effects, while others do not benefit at all, with no way for clinicians to distinguish between likely responders and nonresponders [27]. The identification of responder phenotypes, including a set of predictive biomarkers, would be particularly helpful for asymptomatic or only minimally sleepy OSA patients who will not accept CPAP treatment, unless some other major benefits, such as an improvement in cardiometabolic risk, can be predicted [146].

Regarding comorbidities overall, CPAP has little efficacy in the secondary prevention of cardiovascular and metabolic comorbidities, but the possibility of benefit remains in highly compliant patients and in some patient subgroups that remain poorly defined.

One area of CPAP therapy that is growing in clinical practice is its role as part of the diagnostic pathway to establish the relationship between symptom profile and OSA, especially in highly symptomatic patients with mild OSA $[147,148]$. Lack of symptom improvement in CPAP-compliant patients indicates that reported symptoms may be the result of other factors such as restless legs syndrome or other sleep disturbance. However, symptomatic improvement with CPAP supports the relationship of daytime symptoms such as EDS to OSA and may increase the likelihood of benefit from other therapies such as MADs, although this possibility has not been validated in prospective studies. 
Combined therapeutic strategies for individualised treatment of OSA and comorbidities

Usual antihypertensive agents are less effective in reducing blood pressure in OSA patients, especially at night and in the morning, although recent evidence indicates that morning administration of antihypertensive therapy is superior to evening [149]. Combining CPAP with medications reduces blood pressure in a clinically relevant way in CPAP-compliant patients [130]. A recent meta-analysis revealed that CPAP is associated with a gain in weight of $0.5 \mathrm{~kg}$ compared with control therapy [150]. As many OSA patients are obese, CPAP treatment should be combined with weight loss and recent evidence indicates that baseline oropharyngeal calibre influences the degree of benefit [151, 152]. Lifestyle modifications, including physical activity, through a wide variety of secondary prevention programmes substantially reduced mortality, recurrent heart attacks and blood pressure [153]. In OSA, only one study evaluated CPAP coupled with weight loss, which when combined was superior in improving blood pressure, insulin resistance and lipid profile than either treatment alone [152]. There is a strong rationale for future mechanistic and clinical research into treatment strategies combining CPAP with comprehensive risk factor management, and to identify personalised therapies for addressing cardiovascular and metabolic risk factors of OSA patients.

\section{Opportunities and challenges of telemedicine}

The quality of care a sleep centre provides is mainly determined by individual patient management over the long term, including continuing efforts to maintain optimal CPAP therapy, rapid detection of low compliance and personalised consideration of alternatives to CPAP where appropriate [154, 155]. Telemedicine offers possibilities to diagnose and follow-up OSA patients [156-166], but several open questions, including ethics, data ownership, prescription, storage, usage and reimbursement issues, slow down its implementation [156]. It remains unclear if telemedicine can replace direct patient-nurse contact and/or permit better treatment customisation [167-169]. Critical aspects must be clarified, including the respective role of companies and physicians in patient care, and the risk of unauthorised use of "big data" [170].

Alternative treatment options for OSA

Several alternative treatment options to CPAP and weight loss are available for selected patient populations, which focus on specific target groups and recognise the increasing demand for tailored treatment $[155,171]$. Open questions regarding alternatives to CPAP include the prediction of success, long-term outcomes and health economics [24]. There is an urgent need to define reliable predictors of treatment success and long-term outcomes [172-176].

Mandibular advancement devices

Although less effective in reducing AHI, evidence supports the use of MADs in mild/moderate OSA, even in positional OSA, with associated symptom improvement $[142,177,178]$. In severe cases, MADs are clearly inferior to CPAP [179]. As there is a large variety of MADs, scientific data cannot be translated from one device to another. Standardisation of MADs is needed to guide therapeutic recommendations and comparison of total costs $[145,180]$.

Ablative surgery

Most ablative surgical options cannot be recommended as single interventions and should only be considered in highly selected patients [177, 181-183]. Tonsillectomy, which is a common therapy in paediatric OSA, also benefits adult patients with enlarged tonsils [184]. Maxillomandibular osteotomy appears as effective as CPAP in selected patients refusing conservative treatment [177, 185]. Bariatric surgery may be particularly effective in selected obese OSA patients $[177,186]$.

\section{Positional therapy}

Positional therapy is as effective as CPAP in reducing AHI in positional OSA, but sleep disturbance is a major concern and long-term compliance is poorly documented [187-189]. Devices to assess long-term compliance with positional therapy are needed. Socially disturbing snoring often persists in the nonsupine positions. Substantial nonresponse, partial improvement, patient selection and treatment costs remain significant concerns.

Hypoglossal nerve stimulation

Results from randomised controlled trials of hypoglossal nerve stimulation are promising [190], but data on long-term outcomes are limited, given evidence is based on the same nonrandomised clinical trial (STAR trial), characterised by an unexplained large dropout rate (28\%) after 48 months of follow-up [191194]. Being a responder or nonresponder is partly explained by the resulting tongue protrusion or retrusion after hypoglossal nerve stimulation and related to the stimulation of proximal or more distal nerval branches [195]. Only a minority of patients are eligible for this intervention [196]. There is a need 
for a more precise description of treatment responders based on outcome parameters equivalent to CPAP and for a better understanding of the underlying pathophysiology relating to treatment response [197, 198]. The use of predictor techniques for hypoglossal stimulation response (e.g. drug-induced sleep endoscopy) is not supported by conclusive prospective studies.

Pharmacological therapy

Pharmacological therapy for OSA has shown disappointing results in earlier studies, but pharmacotherapy for selected patients is still under debate [199]. Pharmacological therapy typically targets certain aspects of pathophysiology such as pharyngeal collapsibility, obesity, arousal threshold and loop gain, which supports a personalised approach to treatment [15, 200-202]. Liraglutide is the first pharmaceutical compound having an indication for OSA, by targeting weight reduction in selected populations, although its effect is weak, resulting in a decline in AHI of only 30\% [203]. Desipramine has been demonstrated to reduce pharyngeal collapsibility in healthy subjects during sleep [204] and to lower AHI in selected OSA patients [205], but its use is hampered by significant side-effects. The combination of supplemental oxygen and a hypnotic has been reported to benefit OSA patients with mild/moderate pharyngeal collapsibility [206], presumably by beneficial effects on arousal and loop gain, and could be considered in highly selected patients where insomnia is a prominent feature. Hypnotic use in such patients needs to be carefully considered, given the adverse events associated with hypnotics (fall risk, memory impairment, progression of OSA and the fact that most patients never stop them). Oxygen supplementation may be considered in patients with persisting hypoxaemia despite CPAP therapy, which has been reported to improve perceived physical functioning [207]. Finally, a recent report indicates benefit from acetazolamide therapy in OSA and comorbid hypertension with reductions in both AHI and blood pressure levels [208].

\section{Integrated approach to patient management}

While CPAP remains the treatment of choice for most patients with moderate/severe OSA, alternative and/or additional treatment options can be considered, depending on the clinical and pathophysiological phenotypic traits $[15,209]$. Clinical variables include body mass index, EDS, insomnia, upper airway anatomical factors (e.g. micrognathia or adenotonsillar hypertrophy), in addition to comorbidities, especially arterial hypertension. Pathophysiological variables include upper airway collapsibility and dilator muscle function, arousal threshold, and loop gain. Where upper airway collapsibility predominates, CPAP is the most appropriate management option, but where other factors play a major role, additional or alternative treatment options could be considered, such as pharmacological therapy or hypoglossal nerve stimulation (where upper airway dilating muscle response is inadequate) and other pharmacological agents with oxygen supplementation (where arousal threshold and loop gain play a significant role). Surgical approaches can be considered where a correctable anatomical abnormality is identified and in bariatric surgery for major obesity. A schematic illustration of the complex role of phenotypes in management considerations of OSA is given in figure 4. Categorising and treating patients based on clinical and pathophysiological phenotypic traits is an attractive theoretical concept, but nevertheless, very little data are available supporting such an approach in daily practice.

Priorities for future research

- Evaluation of predictors of treatment outcome.

- Evaluation of the concept of "diagnostic treatment".

- Evaluation of phenotypes predisposing to benefit from surgery, MADs and drugs.

- Identification of potential benefits of surgical interventions.

- Inclusion of objective evaluation of snoring and cardiovascular outcome parameters in outcome studies.

- Identification of treatment options focusing on pathophysiological aspects (loop gain and arousal threshold) and sleep stages (reduction in rapid eye movement sleep).

- Evaluation of the additional long-term effect of oxygen in insufficient positive airway pressure response.

- Evaluation of the combination of OSA treatment with other interventions.

- Validation of biomarkers predicting CPAP responses.

- Identification of the most appropriate therapeutic strategy in OSA patients without symptoms, given their limited acceptance of CPAP.

\section{Conclusions}

The heterogeneity of breathing disturbances associated with OSA, daytime or night-time symptoms and end-organ damage advocates against a simple approach focusing mainly on AHI. There is an urgent need for the definition of phenotypes, based on polysomnographic, clinical and outcome parameters. The 


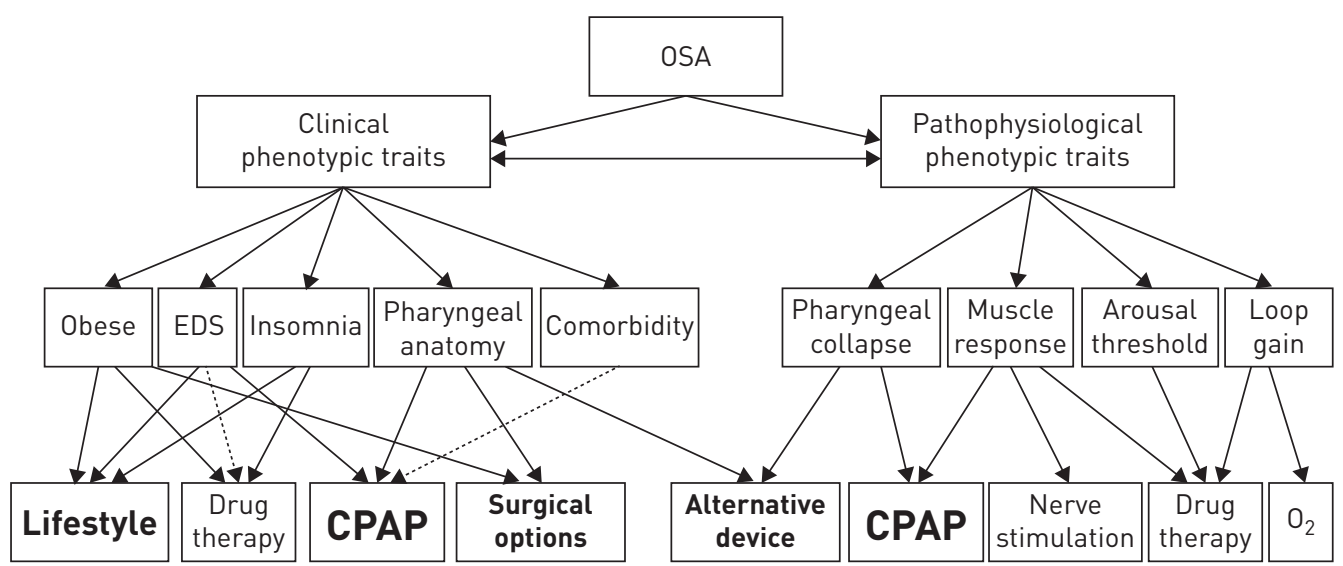

FIGURE 4 Role of clinical and pathophysiological traits in treatment selection for obstructive sleep apnoea (OSA). Schematic illustration of the potential impact of phenotypic traits on treatment options in OSA. Individual treatment options are given different emphasis, based on their relative importance in the overall management of OSA, although the evidence to support the use of drug therapy and $\mathrm{O}_{2}$ supplementation in modifying arousal threshold and loop gain is limited. EDS: excessive daytime sleepiness; CPAP: continuous positive airway pressure; $\mathrm{O}_{2}$ : oxygen.

diagnostic work-up should integrate this multifactorial approach, define severity, not only based on AHI, but include EDS, NPDs (e.g. cognitive impairment and depression), associated sleep disturbances (e.g. insomnia), consequences and prognosis. This may facilitate an individualised and critical use of positive airway pressure and emerging new therapies. These considerations indicate that adequate training and expertise are required of clinicians treating patients with OSA, and support the implementation of speciality training and certification in sleep medicine for sleep practitioners, as advocated by both the ERS and ESRS [210, 211].

Conflict of interest: W. Randerath reports grants and personal fees (travel grants and speaking fees) from Heinen \& Löwenstein, Weinmann, ResMed, Inspire and Philips Respironics, outside the submitted work. L. Ferini-Strambi reports personal fees from Philips Respironics (honoraria for a lecture and for chairing a scientific meeting in 2015-2016, none of which involved promotion of company products and there was no influence on lecture content) and ResMed (honoraria for participating in an advisory board), outside the submitted work. R. Farre reports other fees from ResMed (a contract between the company and the University of Barcelona (Fundació Bosch Gimpera) for assessing automatic CPAP devices in a bench test) and other fees from ANTADIR (a contract between the company and the University of Barcelona (Fundació Bosch Gimpera) for assessing automatic CPAP devices in a bench test), outside the submitted work. L. Grote reports grants and personal fees (speakers' bureau) from ResMed and Philips, personal fees from Breas Mecical (consultancy), personal fees and nonfinancial support from Heinen \& Löwenstein (consultancy), and grants from the ERS, during the conduct of the study; and personal fees (speakers' bureau) from AstraZeneca, outside the submitted work. In addition, L. Grote has a patent on pharmacological treatment of OSA pending. J. Hedner has together with the ESADA study group received grants from ResMed and Philips Respironics to enable the ESADA database. He has also received personal speaker bureau fees from AstraZeneca, Takeda and Bayer. M. Kohler reports personal fees from Bayer (advisor fees), outside the submitted work. J-L. Pepin reports unrestricted research grants from Philips, ResMed, Fisher \& Paykel, Fondation de la recherche medicale, Direction de la recherche Clinique du CHU de Grenoble and Fond de dotation "Agir pour les maladies chroniques", and personal fees (travel grants and lecture fees) from Perimetre, Philips, Fisher \& Paykel, ResMed, AstraZeneka, SEFAM, Agiradom and Teva, during the conduct of the study. J. Verbraecken reports other fees (sponsoring courses) from Koninklijke Philips Respironics, ResMed, Fisher \& Paykel, NightBalance, Heinen \& Löwenstein, AirLiquide, Accuramed, OSG, Medidis, MediqTefa, Wave Medical, Vivisol, TotalCare, SomnoMed, UCB Pharma, Olympus Belgium, Takeda, Masimo and Linde Healthcare, outside the submitted work. W.T. McNicholas reports personal fees from Philips Respironics (honoraria for lectures, but none of these lectures related to promotion of company products and the lecture content was not in any way influenced by the company), outside the submitted work.

Support statement: The Think Tank meeting was funded by unrestricted grants from the European Sleep Foundation (former Alpine Sleep Summer School) Sleep and Health (Lugano, Switzerland), Respironics GmbH (Herrsching, Germany) and ResMed EPN Ltd (Vimercate, Italy). Role of the funding source: none.

\section{References}

1 Randerath W, Verbraecken J, Andreas S, et al. Definition, discrimination, diagnosis and treatment of central breathing disturbances during sleep. Eur Respir J 2017; 49; 1600959.

2 Levy P, Kohler M, McNicholas WT, et al. Obstructive sleep apnoea syndrome. Nat Rev Dis Primers 2015; 1: 15015.

3 Deegan PC, McNicholas WT. Predictive value of clinical features for the obstructive sleep apnoea syndrome. Eur Respir J 1996; 9: 117-124. 
McNicholas WT, Bonsignore MR. Sleep apnoea as an independent risk factor for cardiovascular disease: current evidence, basic mechanisms and research priorities. Eur Respir J 2007; 29: 156-178.

Bonsignore MR, Borel AL, Machan E, et al. Sleep apnoea and metabolic dysfunction. Eur Respir Rev 2013; 22: 353-364.

McEvoy RD, Antic NA, Heeley E, et al. CPAP for prevention of cardiovascular events in obstructive sleep apnea. N Engl J Med 2016; 375: 919-931.

Peker Y, Glantz H, Eulenburg C, et al. Effect of positive airway pressure on cardiovascular outcomes in coronary artery disease patients with nonsleepy obstructive sleep apnea. The RICCADSA randomized controlled trial. Am J Respir Crit Care Med 2016; 194: 613-620.

$\mathrm{Yu}$ J, Zhou Z, McEvoy RD, et al. Association of positive airway pressure with cardiovascular events and death in adults with sleep apnea: a systematic review and meta-analysis. JAMA 2017; 318: 156-166.

Carberry JC, Amatoury J, Eckert DJ. Personalized management approach for OSA. Chest 2018; 153: 744-755.

Bassetti CL, Ferini-Strambi L, Brown S, et al. Neurology and psychiatry: waking up to opportunities of sleep. State of the art and clinical/research priorities for the next decade. Eur J Neurol 2015; 22: 1337-1354.

German Society for Sleep Research and Sleep Medicine. S3-Leitlinie Nicht erholsamer Schlaf/Schlafstörungen. Kapitel "Schlafbezogene Atmungsstörungen". [S3-Guideline: non-restorative sleep. Chapter: sleep related breathing disturbances.] Somnologie 2017; 20: Suppl. S2, S97-S180.

Kapur VK, Auckley DH, Chowdhuri S, et al. Clinical practice guideline for diagnostic testing for adult obstructive sleep apnea: an American Academy of Sleep Medicine clinical practice guideline. J Clin Sleep Med 2017; 13: 479-504.

Escourrou P, Grote L, Penzel T, et al. The diagnostic method has a strong influence on classification of obstructive sleep apnea. J Sleep Res 2015; 24: 730-738.

Edwards BA, Wellman A, Sands SA, et al. Obstructive sleep apnea in older adults is a distinctly different physiological phenotype. Sleep 2014; 37: 1227-1236.

Owens RL, Edwards BA, Eckert DJ, et al. An integrative model of physiological traits can be used to predict obstructive sleep apnea and response to non positive airway pressure therapy. Sleep 2015; 38: 961-970.

Chen H, Aarab G, de Ruiter MH, et al. Three-dimensional imaging of the upper airway anatomy in obstructive sleep apnea: a systematic review. Sleep Med 2016; 21: 19-27.

Bailly S, Destors M, Grillet Y, et al. Obstructive sleep apnea: a cluster analysis at time of diagnosis. PLoS One 2016; 11: e0157318.

Lacedonia D, Carpagnano GE, Sabato R, et al. Characterization of obstructive sleep apnea-hypopnea syndrome (OSA) population by means of cluster analysis. J Sleep Res 2016; 25: 724-730.

Vavougios GD, George DG, Pastaka C, et al. Phenotypes of comorbidity in OSAS patients: combining categorical principal component analysis with cluster analysis. J Sleep Res 2016; 25: 31-38.

Saaresranta T, Hedner J, Bonsignore MR, et al. Clinical phenotypes and comorbidity in European sleep apnoea patients. PLoS One 2016; 11: e0163439.

Gagnadoux F, Le Vaillant M, Paris A, et al. Relationship between OSA clinical phenotypes and CPAP treatment outcomes. Chest 2016; 149: 288-290.

Ye L, Pien GW, Ratcliffe SJ, et al. The different clinical faces of obstructive sleep apnoea: a cluster analysis. Eur Respir J 2014; 44: 1600-1607.

Biomarkers Definitions Working Group. Biomarkers and surrogate endpoints: preferred definitions and conceptual framework. Clin Pharmacol Ther 2001; 69: 89-95.

Pack AI. Application of personalized, predictive, preventative, and participatory (P4) medicine to obstructive sleep apnea. A roadmap for improving care? Ann Am Thorac Soc 2016; 13: 1456-1467.

Khalyfa A, Gileles-Hillel A, Gozal D. The challenges of precision medicine in obstructive sleep apnea. Sleep Med Clin 2016; 11: 213-226.

Jullian-Desayes I, Joyeux-Faure M, Tamisier R, et al. Impact of obstructive sleep apnea treatment by continuous positive airway pressure on cardiometabolic biomarkers: a systematic review from sham CPAP randomized controlled trials. Sleep Med Rev 2015; 21: 23-38.

Sanchez-de-la-Torre M, Khalyfa A, Sanchez-de-la-Torre A, et al. Precision medicine in patients with resistant hypertension and obstructive sleep apnea: blood pressure response to continuous positive airway pressure treatment. J Am Coll Cardiol 2015; 66: 1023-1032.

Schwarz EI, Martinez-Lozano Sinues P, Bregy L, et al. Effects of CPAP therapy withdrawal on exhaled breath pattern in obstructive sleep apnoea. Thorax 2016; 71: 110-117.

Flemons WW, Buysse D, Redline S, et al. Sleep-related breathing disorders in adults: recommendations for syndrome definition and measurement techniques in clinical research. Sleep 1999; 22: 667-689.

Arnardottir ES, Bjornsdottir E, Olafsdottir KA, et al. Obstructive sleep apnoea in the general population: highly prevalent but minimal symptoms. Eur Respir J 2016; 47: 194-202.

Heinzer R, Vat S, Marques-Vidal P, et al. Prevalence of sleep-disordered breathing in the general population: the HypnoLaus study. Lancet Respir Med 2015; 3: 310-318.

Kuna ST, Badr MS, Kimoff RJ, et al. An official ATS/AASM/ACCP/ERS workshop report: research priorities in ambulatory management of adults with obstructive sleep apnea. Proc Am Thorac Soc 2011; 8: 1-16.

Tkacova R, McNicholas WT, Javorsky M, et al. Nocturnal intermittent hypoxia predicts prevalent hypertension in the European Sleep Apnoea Database cohort study. Eur Respir J 2014; 44: 931-941.

Seif F, Patel SR, Walia HK, et al. Obstructive sleep apnea and diurnal nondipping hemodynamic indices in patients at increased cardiovascular risk. J Hypertens 2014; 32: 267-275.

McNicholas WT, Bonsignore MR, Levy P, et al. Mild obstructive sleep apnoea: clinical relevance and approaches to management. Lancet Respir Med 2016; 4: 826-834.

Younes M, Soiferman M, Thompson W, et al. Performance of a new portable wireless sleep monitor. J Clin Sleep Med 2017; 13: 245-258.

Guerrero A, Embid C, Isetta V, et al. Management of sleep apnea without high pretest probability or with comorbidities by three nights of portable sleep monitoring. Sleep 2014; 37: 1363-1373.

Berry RB, Budhiraja R, Gottlieb DJ, et al. Rules for scoring respiratory events in sleep: update of the 2007 AASM manual for the scoring of sleep and associated events. J Clin Sleep Med 2012; 8: 597-619. 
Ruehland WR, Rochford PD, O'Donoghue FJ, et al. The new AASM criteria for scoring hypopneas: impact on the apnea hypopnea index. Sleep 2009; 32: 150-157.

Campos-Rodriguez F, Martínez-García MA, Reyes-Nuñez N, et al. Impact of different hypopnea definitions on obstructive sleep apnea severity and cardiovascular mortality risk in women and elderly individuals. Sleep Med 2016; 27-28: 54-58.

Kulkas A, Duce B, Leppanen T, et al. Severity of desaturation events differs between hypopnea and obstructive apnea events and is modulated by their duration in obstructive sleep apnea. Sleep Breath 2017; 21: 829-835.

Campos-Rodriguez F, Gonzalez-Martinez M, Sanchez-Armengol A, et al. Effect of continuous positive airway pressure on blood pressure and metabolic profile in women with sleep apnoea. Eur Respir J 2017; 50: 1700257.

Kingshott RN, Sime PJ, Engleman HM, et al. Self assessment of daytime sleepiness: patient versus partner. Thorax 1995; 50: 994-995.

Ustun B, Westover MB, Rudin C, et al. Clinical prediction models for sleep apnea: the importance of medical history over symptoms. J Clin Sleep Med 2016; 12: 161-168.

Crinion SJ, Ryan S, McNicholas WT. Obstructive sleep apnoea as a cause of nocturnal nondipping blood pressure: recent evidence regarding clinical importance and underlying mechanisms. Eur Respir J 2017; 49: 1601818.

Marti-Soler H, Hirotsu C, Marques-Vidal P, et al. The NoSAS score for screening of sleep-disordered breathing: a derivation and validation study. Lancet Respir Med 2016; 4: 742-748.

Arnardottir ES, Gislason T. Quantifying airflow limitation and snoring during sleep. Sleep Med Clin 2016; 11: 421-434.

Dewan NA, Nieto FJ, Somers VK. Intermittent hypoxemia and OSA: implications for comorbidities. Chest 2015; 147: 266-274.

Khoo MCK, Chalacheva P. Model-derived markers of autonomic cardiovascular dysfunction in sleep disordered breathing. Sleep Med Clin 2016; 11: 489-501.

Alshaer H, Fernie GR, Tseng WH, et al. Comparison of in-laboratory and home diagnosis of sleep apnea using a cordless portable acoustic device. Sleep Med 2016; 22: 91-96.

Abbasi J. In-home, over-the-counter sleep apnea sensor on the horizon. JAMA 2017; 317: 2271. patients with sleep apnea. Chest 2014; 145: 1025-1031.

Jacobsen JH, Shi L, Mokhlesi B. Factors associated with excessive daytime sleepiness in patients with severe obstructive sleep apnea. Sleep Breath 2013; 17: 629-635.

Adams RJ, Appleton SL, Vakulin A, et al. Association of daytime sleepiness with obstructive sleep apnoea and comorbidities varies by sleepiness definition in a population cohort of men. Respirology 2016; 21: 1314-1321.

Guaita M, Salamero M, Vilaseca I, et al. The Barcelona Sleepiness Index: a new instrument to assess excessive daytime sleepiness in sleep disordered breathing. J Clin Sleep Med 2015; 11: 1289-1298.

Kendzerska TB, Smith PM, Brignardello-Petersen R, et al. Evaluation of the measurement properties of the Epworth sleepiness scale: a systematic review. Sleep Med Rev 2014; 18: 321-331.

Ohayon MM, Dauvilliers Y, Reynolds CF 3rd. Operational definitions and algorithms for excessive sleepiness in the general population: implications for DSM-5 nosology. Arch Gen Psychiatry 2012; 69: 71-79.

Bhat S, Upadhyay H, DeBari VA, et al. The utility of patient-completed and partner-completed Epworth Sleepiness Scale scores in the evaluation of obstructive sleep apnea. Sleep Breath 2016; 20: 1347-1354.

Pizza F, Contardi S, Mondini S, et al. Simulated driving performance coupled with driver behaviour can predict the risk of sleepiness-related car accidents. Thorax 2011; 66: 725-726.

Pizza F, Contardi S, Mondini S, et al. Daytime sleepiness and driving performance in patients with obstructive sleep apnea: comparison of the MSLT, the MWT, and a simulated driving task. Sleep 2009; 32: 382-391.

Ren R, Li Y, Zhang J, et al. Obstructive sleep apnea with objective daytime sleepiness is associated with hypertension. Hypertension 2016; 68: 1264-1270.

Ronksley PE, Hemmelgarn BR, Heitman SJ, et al. Excessive daytime sleepiness is associated with increased health care utilization among patients referred for assessment of OSA. Sleep 2011; 34: 363-370.

Li Y, Vgontzas AN, Fernandez-Mendoza J, et al. Objective, but not subjective. Sleepiness is associated with inflammation in sleep apnea. Sleep 2017; 40: zsw033.

Wang W, Pan Y, Li Q, et al. Orexin: a potential role in the process of obstructive sleep apnea. Peptides 2013; 42: 48-54.

Arita A, Sasanabe R, Hasegawa R, et al. Risk factors for automobile accidents caused by falling asleep while driving in obstructive sleep apnea syndrome. Sleep Breath 2015; 19: 1229-1234.

Tregear S, Reston J, Schoelles K, et al. Continuous positive airway pressure reduces risk of motor vehicle crash among drivers with obstructive sleep apnea: systematic review and meta-analysis. Sleep 2010; 33: 1373-1380.

Goncalves M, Amici R, Lucas R, et al. Sleepiness at the wheel across Europe: a survey of 19 countries. J Sleep Res 2015; 24: 242-253.

Karimi M, Hedner J, Lombardi C, et al. Driving habits and risk factors for traffic accidents among sleep apnea patients - a European multi-centre cohort study. J Sleep Res 2014; 23: 689-699.

Antonopoulos CN, Sergentanis TN, Daskalopoulou SS, et al. Nasal continuous positive airway pressure (nCPAP) treatment for obstructive sleep apnea, road traffic accidents and driving simulator performance: a meta-analysis. Sleep Med Rev 2011; 15: 301-310.

Abe T, Komada Y, Inoue Y. Short sleep duration, snoring and subjective sleep insufficiency are independent factors associated with both falling asleep and feeling sleepiness while driving. Intern Med 2012; 51: 3253-3260.

Sukhal S, Khalid M, Tulaimat A. Effect of wakefulness-promoting agents on sleepiness in patients with sleep apnea treated with CPAP: a meta-analysis. J Clin Sleep Med 2015; 11: 1179-1186.

Kuan YC, Wu D, Huang KW, et al. Effects of modafinil and armodafinil in patients with obstructive sleep apnea: a meta-analysis of randomized controlled trials. Clin Ther 2016; 38: 874-888.

Bonsignore MR, Randerath W, Riha R, et al. New rules on driver licensing for patients with obstructive sleep apnea: European Union Directive 2014/85/EU. J Sleep Res 2016; 25: 3-4.

Gupta M, Simpson FC. Obstructive sleep apnea and psychiatric disorders: a systematic review. J Clin Sleep Med 2015; 11: 165-175. 

depressive disorder, bipolar disorder and schizophrenia: a systematic review and meta-analysis. J Affect Disord 2016; 197: 259-267.

76 Haba-Rubio J, Marti-Soler H, Tobback N, et al. Sleep characteristics and cognitive impairment in the general population: the HypnoLaus study. Neurology 2017; 88: 463-469.

77 Sforza E, Saint Martin M, Barthelemy JC, et al. Mood disorders in healthy elderly with obstructive sleep apnea: a gender effect. Sleep Med 2016; 19: 57-62.

78 Hermann DM, Bassetti CL. Role of sleep-disordered breathing and sleep-wake disturbances for stroke and stroke recovery. Neurology 2016; 87: 1407-1416.

79 Chen YH, Keller JK, Kang JH, et al. Obstructive sleep apnea and the subsequent risk of depressive disorder: a population-based follow-up study. J Clin Sleep Med 2013; 9: 417-423.

80 Buratti L, Luzzi S, Petrelli C, et al. Obstructive sleep apnea syndrome: an emerging risk factor for dementia. CNS Neurol Disord Drug Targets 2016; 15: 678-682.

81 Ding X, Kryscio RJ, Turner J, et al. Self-reported sleep apnea and dementia risk: findings from the prevention of Alzheimer's Disease with Vitamin E and Selenium Trial. J Am Geriatr Soc 2016; 64: 2472-2478.

82 Lutsey PL, Norby FL, Gottesman RF, et al. Sleep apnea, sleep duration and brain MRI markers of cerebral vascular disease and Alzheimer's disease: the Atherosclerosis Risk in Communities study (ARIC). PLoS One 2016; 11: e0158758.

83 Rosenzweig I, Glasser M, Polsek D, et al. Sleep apnoea and the brain: a complex relationship. Lancet Respir Med 2015; 3: 404-414.

84 Pincherle A, Pace M, Sarasso S, et al. Sleep, preconditioning and stroke. Stroke 2017; 48: 3400-3407.

85 Lavie L. Oxidative stress in obstructive sleep apnea and intermittent hypoxia - revisited - the bad ugly and good: implications to the heart and brain. Sleep Med Rev 2015; 20: 27-45.

86 Snyder B, Shell B, Cunningham JT, et al. Chronic intermittent hypoxia induces oxidative stress and inflammation in brain regions associated with early-stage neurodegeneration. Physiol Rep 2017; 5: e13258.

87 Daulatzai MA. Evidence of neurodegeneration in obstructive sleep apnea: relationship between obstructive sleep apnea and cognitive dysfunction in the elderly. J Neurosci Res 2015; 93: 1778-1794.

88 Yaffe K, Falvey CM, Hoang T. Connections between sleep and cognition in older adults. Lancet Neurol 2014; 13 1017-1028.

89 Canessa N, Castronovo V, Cappa SF, et al. Obstructive sleep apnea: brain structural changes and neurocognitive function before and after treatment. Am J Respir Crit Care Med 2011; 183: 1419-1426.

90 Castronovo V, Scifo P, Castellano A, et al. White matter integrity in obstructive sleep apnea before and after treatment. Sleep 2014; 37: 1465-1475.

91 Kerner NA, Roose SP. Obstructive sleep apnea is linked to depression and cognitive impairment: evidence and potential mechanisms. Am J Geriatr Psychiatry 2016; 24: 496-508.

92 Osorio RS, Gumb T, Pirraglia E, et al. Sleep-disordered breathing advances cognitive decline in the elderly. Neurology 2015; 84: 1964-1971.

93 Edwards C, Mukherjee S, Simpson L, et al. Depressive symptoms before and after treatment of obstructive sleep apnea in men and women. J Clin Sleep Med 2016; 11: 1029-1038.

94 Ferini-Strambi L, Marelli S, Galbiati A, et al. Effects of continuous positive airway pressure on cognition and neuroimaging data in sleep apnea. Int J Psychophysiol 2013; 89: 203-212.

95 Harmell AL, Neikrug AB, Palmer BW, et al. Obstructive sleep apnea and cognition in Parkinson's disease. Sleep Med 2016; 21: 28-34.

96 Neikrug AB, Liu L, Avanzino JA, et al. Continuous positive airway pressure improves sleep and daytime sleepiness in patients with Parkinson disease and sleep apnea. Sleep 2014; 37: 177-185.

97 Gupta MA, Simpson FC, Lyons DC. The effect of treating obstructive sleep apnea with positive airway pressure on depression and other subjective symptoms: a systematic review and meta-analysis. Sleep Med Rev 2016; 28: 55-68.

98 Senaratna CV, Perret JL, Lodge CJ, et al. Prevalence of obstructive sleep apnea in the general population: a systematic review. Sleep Med Rev 2017; 34: 70-81.

99 Farrell PC, Richards G. Recognition and treatment of sleep-disordered breathing: an important component of chronic disease management. J Transl Med 2017; 15: 114.

100 Vgontzas AN, Gaines J, Ryan S, et al. CrossTalk proposal: metabolic syndrome causes sleep apnoea. J Physiol 2016; 594: 4687-4690.

101 Anothaisintawee T, Reutrakul S, Van Cauter E, et al. Sleep disturbances compared to traditional risk factors for diabetes development: systematic review and meta-analysis. Sleep Med Rev 2016; 30: 11-24.

102 Kent BD, Grote L, Ryan S, et al. Diabetes mellitus prevalence and control in sleep-disordered breathing: the European Sleep Apnea Cohort (ESADA) study. Chest 2014; 146: 982-990.

103 Nobili V, Cutrera R, Liccardo D, et al. Obstructive sleep apnea syndrome affects liver histology and inflammatory cell activation in pediatric nonalcoholic fatty liver disease, regardless of obesity/insulin resistance. Am J Respir Crit Care Med 2014; 189: 66-76.

104 Sundaram SS, Halbower A, Pan Z, et al. Nocturnal hypoxia-induced oxidative stress promotes progression of pediatric non-alcoholic fatty liver disease. J Hepatol 2016; 65: 560-569.

105 Sookoian S, Pirola CJ. Obstructive sleep apnea is associated with fatty liver and abnormal liver enzymes: a meta-analysis. Obes Surg 2013; 23: 1815-1825.

106 Aron-Wisnewsky J, Clement K, Pepin JL. Nonalcoholic fatty liver disease and obstructive sleep apnea. Metabolism 2016; 65: 1124-1135.

107 Aron-Wisnewsky J, Minville C, Tordjman J, et al. Chronic intermittent hypoxia is a major trigger for non-alcoholic fatty liver disease in morbid obese. J Hepatol 2012; 56: 225-233.

108 Kendzerska T, Gershon AS, Hawker G, et al. Obstructive sleep apnea and risk of cardiovascular events and all-cause mortality: a decade-long historical cohort study. PLoS Med 2014; 11: e1001599.

109 Jennum P, Kjellberg J. Health, social and economical consequences of sleep-disordered breathing: a controlled national study. Thorax 2011; 66: 560-566.

110 Kim MY, Jo EJ, Kang SY, et al. Obstructive sleep apnea is associated with reduced quality of life in adult patients with asthma. Ann Allergy Asthma Immunol 2013; 110: 253-257. 
111 Luyster FS, Teodorescu M, Bleecker E, et al. Sleep quality and asthma control and quality of life in non-severe and severe asthma. Sleep Breath 2012; 16: 1129-1137.

112 Kong DL, Qin Z, Shen H, et al. Association of obstructive sleep apnea with asthma: a meta-analysis. Sci Rep 2017; 7: 4088

113 Teodorescu M, Consens FB, Bria WF, et al. Correlates of daytime sleepiness in patients with asthma. Sleep Med 2006; 7: 607-613

114 Teodorescu M, Polomis DA, Hall SV, et al. Association of obstructive sleep apnea risk with asthma control in adults. Chest 2010; 138: 543-550.

115 Broytman O, Braun RK, Morgan BJ, et al. Effects of chronic intermittent hypoxia on allergen-induced airway inflammation in rats. Am J Respir Cell Mol Biol 2015; 52: 162-170.

116 Becerra MB, Becerra BJ, Teodorescu M. Healthcare burden of obstructive sleep apnea and obesity among asthma hospitalizations: results from the U.S.-based Nationwide Inpatient Sample. Respir Med 2016; 117: 230-236.

117 Mermigkis C, Bouloukaki I, Schiza SE. Sleep as a new target for improving outcomes in idiopathic pulmonary fibrosis. Chest 2017; 152: 1327-1338.

118 Bosi M, Milioli G, Fanfulla F, et al. OSA and prolonged oxygen desaturation during sleep are strong predictors of poor outcome in IPF. Lung 2017; 195: 643-651.

119 Braun RK, Broytman O, Braun FM, et al. Chronic intermittent hypoxia worsens bleomycin-induced lung fibrosis in rats. Respir Physiol Neurobiol 2017; in press [https://doi.org/10.1016/j.resp.2017.04.010].

120 Lederer DJ, Jelic S, Basner RC, et al. Circulating KL-6, a biomarker of lung injury, in obstructive sleep apnea. Eur Respir J 2009; 33: 793-796.

121 Lee RN, Kelly E, Nolan G, et al. Disordered breathing during sleep and exercise in idiopathic pulmonary fibrosis and the role of biomarkers. QJM 2015; 108: 315-323.

122 McNicholas WT. COPD-OSA overlap syndrome: evolving evidence regarding epidemiology, clinical consequences, and management. Chest 2017; 152: 1318-1326.

123 Malhotra A, Schwartz AR, Schneider H, et al. Research priorities in pathophysiology for sleep-disordered breathing in patients with chronic obstructive pulmonary disease. An official American Thoracic Society research statement. Am J Respir Crit Care Med 2018; 197: 289-299.

124 Adler D, Pepin JL, Dupuis-Lozeron E, et al. Comorbidities and subgroups of patients surviving severe acute hypercapnic respiratory failure in the intensive care unit. Am J Respir Crit Care Med 2017; 196: 200-207.

125 Marin JM, Soriano JB, Carrizo SJ, et al. Outcomes in patients with chronic obstructive pulmonary disease and obstructive sleep apnea: the overlap syndrome. Am J Respir Crit Care Med 2010; 182: 325-331.

126 Zhang XB, Peng LH, Lyu Z, et al. Obstructive sleep apnoea and the incidence and mortality of cancer: a meta-analysis. Eur J Cancer Care 2017; 26: e12427.

127 Campos-Rodriguez F, Martinez-Garcia MA, Martinez M, et al. Association between obstructive sleep apnea and cancer incidence in a large multicenter Spanish cohort. Am J Respir Crit Care Med 2013; 187: 99-105.

128 Gozal D, Farre R, Nieto FJ. Obstructive sleep apnea and cancer: epidemiologic links and theoretical biological constructs. Sleep Med Rev 2016; 27: 43-55.

129 Thunstrom E, Manhem K, Rosengren A, et al. Blood pressure response to losartan and continuous positive airway pressure in hypertension and obstructive sleep apnea. Am J Respir Crit Care Med 2016; 193: 310-320.

130 Pepin JL, Tamisier R, Barone-Rochette G, et al. Comparison of continuous positive airway pressure and valsartan in hypertensive patients with sleep apnea. Am J Respir Crit Care Med 2010; 182: 954-960.

131 Miller JD, Aronis KN, Chrispin J, et al. Obesity, exercise, obstructive sleep apnea, and modifiable atherosclerotic cardiovascular disease risk factors in atrial fibrillation. J Am Coll Cardiol 2015; 66: 2899-2906.

132 Reutrakul S, Mokhlesi B. Obstructive sleep apnea and diabetes: a state of the art review. Chest 2017; 152: 1070-1086.

133 Khayat R, Jarjoura D, Porter K, et al. Sleep disordered breathing and post-discharge mortality in patients with acute heart failure. Eur Heart J 2015; 36: 1463-1469.

134 McNicholas WT. Chronic obstructive pulmonary disease and obstructive sleep apnoea - the overlap syndrome. J Thorac Dis 2016; 8: 236-242.

135 Cammaroto G, Galletti C, Galletti F, et al. Mandibular advancement devices vs nasal-continuous positive airway pressure in the treatment of obstructive sleep apnoea. Systematic review and meta-analysis. Med Oral Patol Oral Cir Bucal 2017; 22: e417-e424.

136 Kuhn E, Schwarz EI, Bratton DJ, et al. Effects of CPAP and mandibular advancement devices on health-related quality of life in OSA: a systematic review and meta-analysis. Chest 2017; 151: 786-794.

137 Bratton DJ, Gaisl T, Wons AM, et al. CPAP vs mandibular advancement devices and blood pressure in patients with obstructive sleep apnea: a systematic review and meta-analysis. JAMA 2015; 314: 2280-2293.

138 Martinez-Garcia MA, Capote F, Campos-Rodriguez F, et al. Effect of CPAP on blood pressure in patients with obstructive sleep apnea and resistant hypertension: the HIPARCO randomized clinical trial. JAMA 2013; 310: 2407-2415.

139 Salord N, Fortuna AM, Monasterio C, et al. A randomized controlled trial of continuous positive airway pressure on glucose tolerance in obese patients with obstructive sleep apnea. Sleep 2016; 39: 35-41.

140 Martinez-Ceron E, Barquiel B, Bezos AM, et al. Effect of continuous positive airway pressure on glycemic control in patients with obstructive sleep apnea and type 2 diabetes. A randomized clinical trial. Am J Respir Crit Care Med 2016; 194: 476-485.

141 Mokhlesi B, Hagen EW, Finn LA, et al. Obstructive sleep apnoea during REM sleep and incident non-dipping of nocturnal blood pressure: a longitudinal analysis of the Wisconsin Sleep Cohort. Thorax 2015; 70: 1062-1069.

142 Marklund M, Verbraecken J, Randerath W. Non-CPAP therapies in obstructive sleep apnoea: mandibular advancement device therapy. Eur Respir J 2012; 39: 1241-1247.

143 Bloch KE, Huber F, Furian M, et al. Autoadjusted versus fixed CPAP for obstructive sleep apnoea: a multicentre, randomised equivalence trial. Thorax 2018; 73: 174-184.

144 Pepin JL, Tamisier R, Baguet JP, et al. Fixed-pressure CPAP versus auto-adjusting CPAP: comparison of efficacy on blood pressure in obstructive sleep apnoea, a randomised clinical trial. Thorax 2016; 71: 726-733.

145 Marklund M. Update on oral appliance therapy for OSA. Curr Sleep Med Rep 2017; 3: 143-151.

146 McEvoy RD, Michael MZ. Measuring blood microRNAs to provide personalized advice to sleep apnea patients with resistant hypertension: dreaming the future. J Am Coll Cardiol 2015; 66: 1033-1035. 


\section{on symptom}

154 Hilbert J, Y 37: 138-147.

155 Bonsignore MR, Suarez Giron MC, Marrone O, et al. Personalised medicine in sleep respiratory disorders: focus on obstructive sleep apnoea diagnosis and treatment. Eur Respir Rev 2017; 26: 170069.

156 Verbraecken J. Telemedicine applications in sleep disordered breathing: thinking out of the box. Sleep Med Clin 2016; 11: 445-459.

157 Wilson SR, Cram P. Another sobering result for home telehealth - and where we might go next. Arch Intern Med 2012; 172: 779-780.

158 Ambrosino N, Vitacca M, Dreher M, et al. Tele-monitoring of ventilator-dependent patients: a European Respiratory Society Statement. Eur Respir J 2016; 48: 648-663.

159 Farre R, Navajas D, Montserrat JM. Is telemedicine a key tool for improving continuous positive airway pressure adherence in patients with sleep apnea? Am J Respir Crit Care Med 2018; 197: 12-14.

160 Mendelson M, Vivodtzev I, Tamisier R, et al. CPAP treatment supported by telemedicine does not improve blood pressure in high cardiovascular risk OSA patients: a randomized, controlled trial. Sleep 2014; 37: 1863-1870.

161 Ruotsalainen P. Privacy and security in teleradiology. Eur J Radiol 2010; 73: 31-35.

162 Dierks C. Legal aspects of telepathology. Anal Cell Pathol 2000; 21: 97-99.

163 Zannad F, Maugendre P, Audry A, et al. Telemedicine: what framework, what levels of proof, implementation rules. Therapie 2014; 69: 339-354.

164 Biselli P, Grossman PR, Kirkness JP, et al. The effect of increased lung volume in chronic obstructive pulmonary disease on upper airway obstruction during sleep. J Appl Physiol 2015; 119: 266-271.

165 Bräunlich J, Beyer D, Mai D, et al. Effects of nasal high flow on ventilation in volunteers, COPD and idiopathic pulmonary fibrosis patients. Respiration 2013; 85: 319-325.

166 Wang Y, Hu K, Liu K, et al. Obstructive sleep apnea exacerbates airway inflammation in patients with chronic obstructive pulmonary disease. Sleep Med 2015; 16: 1123-1130.

167 Lugo V, Villanueva JA, Garmendia O, et al. The role of telemedicine in obstructive sleep apnea management. Expert Rev Respir Med 2017; 11: 699-709.

168 Isetta V, Leon C, Torres $\mathrm{M}$, et al. Telemedicine-based approach for obstructive sleep apnea management: building evidence. Interact J Med Res 2014; 3: e6.

169 Anttalainen U, Melkko S, Hakko S, et al. Telemonitoring of CPAP therapy may save nursing time. Sleep Breath 2016; 20: 1209-1215.

170 Isetta V, Negrin MA, Monasterio C, et al. A Bayesian cost-effectiveness analysis of a telemedicine-based strategy for the management of sleep apnoea: a multicentre randomised controlled trial. Thorax 2015; 70: 1054-1061.

171 Eckert DJ. Phenotypic approaches to obstructive sleep apnoea - new pathways for targeted therapy. Sleep Med Rev 2018; 37: 45-59.

172 Okuno K, Pliska BT, Hamoda M, et al. Prediction of oral appliance treatment outcomes in obstructive sleep apnea: a systematic review. Sleep Med Rev 2016; 30: 25-33.

173 Verbruggen A, Vroegop A, Dieltjens M, et al. Predicting therapeutic outcome of mandibular advancement device treatment in obstructive sleep apnoea (PROMAD): study design and baseline characteristics. J Dent Sleep Med 2016; 3: 119-138.

174 Saffer F, Lubianca Neto JF, Rosing C, et al. Predictors of success in the treatment of obstructive sleep apnea syndrome with mandibular repositioning appliance: a systematic review. Int Arch Otorhinolaryngol 2015; 19: $80-85$.

175 Bharathi MB, Rajendra Prasad J, Satish K. Drug-induced sleep endoscopy as a selection tool for surgical management of obstructive sleep apnoea syndrome: our personal experience. Indian J Otolaryngol Head Neck Surg 2017; 69: 313-318.

176 Blumen M, Bequignon E, Chabolle F. Drug-induced sleep endoscopy: a new gold standard for evaluating OSAS? Part I: technique. Eur Ann Otorhinolaryngol Head Neck Dis 2017; 134: 101-107.

177 Randerath WJ, Verbraecken J, Andreas S, et al. Non-CPAP therapies in obstructive sleep apnoea. Eur Respir J 2011; 37: 1000-1028.

178 Takaesu Y, Tsuiki S, Kobayashi M, et al. Mandibular advancement device as a comparable treatment to nasal continuous positive airway pressure for positional obstructive sleep apnea. J Clin Sleep Med 2016; 12: 1113-1119.

179 O'Donoghue F, McDonald C. Review: CPAP improves QoL in obstructive sleep apnea; effects not as clear for mandibular advancement devices. Ann Intern Med 2017; 167: JC18.

180 Sharples L, Glover M, Clutterbuck-James A, et al. Clinical effectiveness and cost-effectiveness results from the randomised controlled Trial of Oral Mandibular Advancement Devices for Obstructive sleep apnoea-hypopnoea (TOMADO) and long-term economic analysis of oral devices and continuous positive airway pressure. Health Technol Assess 2014; 18: 1-296.

181 Browaldh N, Nerfeldt P, Lysdahl M, et al. SKUP3 randomised controlled trial: polysomnographic results after uvulopalatopharyngoplasty in selected patients with obstructive sleep apnoea. Thorax 2013; 68: 846-853. 
Camacho M, Nesbitt NB, Lambert E, et al. Laser-assisted uvulopalatoplasty for obstructive sleep apnea: a systematic review and meta-analysis. Sleep 2017; 40: zsx004.

Verse T, Dreher A, Heiser C, et al. ENT-specific therapy of obstructive sleep apnoea in adults: a revised version of the previously published German S2e guideline. Sleep Breath 2016; 20: 1301-1311.

Camacho M, Li D, Kawai M, et al. Tonsillectomy for adult obstructive sleep apnea: a systematic review and meta-analysis. Laryngoscope 2016; 126: 2176-2186.

Noller MW, Guilleminault C, Gouveia CJ, et al. Mandibular advancement for adult obstructive sleep apnea: a systematic review and meta-analysis. J Craniomaxillofac Surg 2017; 45: 2035-2040.

de Raff CAL, Gorter-Stam MAW, de Vries N, et al. Perioperative management of obstructive sleep apnea in bariatric surgery: a consensus guideline. Surg Obes Relat Dis 2017; 13: 1095-1109.

Ravesloot MJL, White D, Heinzer R, et al. Efficacy of the new generation of devices for positional therapy for patients with positional obstructive sleep apnea: a systematic review of the literature and meta-analysis. $J$ Clin Sleep Med 2017; 13: 813-824.

Ravesloot MJ, van Maanen JP, Dun L, et al. The undervalued potential of positional therapy in position-dependent snoring and obstructive sleep apnea - a review of the literature. Sleep Breath 2013; 17: 39-49. van Maanen JP, de Vries N. Long-term effectiveness and compliance of positional therapy with the sleep position trainer in the treatment of positional obstructive sleep apnea syndrome. Sleep 2014; 37: 1209-1215.

Strollo PJ Jr, Gillespie MB, Soose RJ, et al. Upper airway stimulation for obstructive sleep apnea: durability of the treatment effect at 18 months. Sleep 2015; 38: 1593-1598.

Soose RJ, Woodson BT, Gillespie MB, et al. Upper airway stimulation for obstructive sleep apnea: self-reported outcomes at 24 months. J Clin Sleep Med 2016; 12: 43-48.

Woodson BT, Soose RJ, Gillespie MB, et al. Three-year outcomes of cranial nerve stimulation for obstructive sleep apnea: the STAR trial. Otolaryngol Head Neck Surg 2016; 154: 181-188.

Gillespie MB, Soose RJ, Woodson BT, et al. Upper airway stimulation for obstructive sleep apnea: patient-reported outcomes after 48 months of follow-up. Otolaryngol Head Neck Surg 2017; 156: 765-771.

Mwenge GB, Rombaux P, Dury M, et al. Targeted
1 -year pilot study. Eur Respir J 2013; 41: 360-367.

Vanderveken OM, Beyers J, Op de Beeck S, et al. Development of a clinical pathway and technical aspects of upper airway stimulation therapy for obstructive sleep apnea. Front Neurosci 2017; 11: 523.

Strollo PJ Jr, Soose RJ, Maurer JT, et al. Upper-airway stimulation for obstructive sleep apnea. N Engl J Med 2014; 370: 139-149.

Safiruddin F, Vanderveken OM, de Vries N, et al. Effect of upper-airway stimulation for obstructive sleep apnoea on airway dimensions. Eur Respir J 2015; 45: 129-138.

Ong AA, Murphey AW, Nguyen SA, et al. Efficacy of upper airway stimulation on collapse patterns observed during drug-induced sedation endoscopy. Otolaryngol Head Neck Surg 2016; 154: 970-977.

White DP. Pharmacologic approaches to the treatment of obstructive sleep apnea. Sleep Med Clin 2016; 11: $203-212$.

Eckert DJ, White DP, Jordan AS, et al. Defining phenotypic causes of obstructive sleep apnea. Identification of novel therapeutic targets. Am J Respir Crit Care Med 2013; 188: 996-1004.

Edwards BA, Sands SA, Eckert DJ, et al. Acetazolamide improves loop gain but not the other physiological traits causing obstructive sleep apnoea. J Physiol 2012; 590: 1199-1211.

Eckert DJ, Malhotra A, Wellman A, et al. Trazodone increases the respiratory arousal threshold in patients with obstructive sleep apnea and a low arousal threshold. Sleep 2014; 37: 811-819.

Blackman A, Foster GD, Zammit G, et al. Effect of liraglutide $3.0 \mathrm{mg}$ in individuals with obesity and moderate or severe obstructive sleep apnea: the SCALE Sleep Apnea randomized clinical trial. Int J Obes 2016; 40: 1310-1319. Taranto-Montemurro L, Edwards BA, Sands SA, et al. Desipramine increases genioglossus activity and reduces upper airway collapsibility during non-REM sleep in healthy subjects. Am J Respir Crit Care Med 2016; 194: 878-885.

5 Taranto-Montemurro L, Sands SA, Edwards BA, et al. Desipramine improves upper airway collapsibility and reduces OSA severity in patients with minimal muscle compensation. Eur Respir J 2016; 48: 1340-1350.

Edwards BA, Sands SA, Owens RL, et al. The combination of supplemental oxygen and a hypnotic markedly improves obstructive sleep apnea in patients with a mild to moderate upper airway collapsibility. Sleep 2016; 39: 1973-1983.

Gottlieb DJ, Punjabi NM, Mehra R, et al. CPAP versus oxygen in obstructive sleep apnea. N Engl J Med 2014; 370: 2276-2285.

Eskandari D, Zou D, Grote L, et al. Acetazolamide reduces blood pressure and sleep-disordered breathing in patients with hypertension and obstructive sleep apnea: a randomized controlled trial. J Clin Sleep Med 2018; 14: 309-317.

Edwards BA, Andara C, Landry S, et al. Upper-airway collapsibility and loop gain predict the response to oral appliance therapy in patients with obstructive sleep apnea. Am J Respir Crit Care Med 2016; $194: 1413-1422$.

Mitchell S, Simonds A, Andreas S, et al. Introducing a core curriculum for respiratory sleep practitioners. Breathe 2015; 11: 50-56.

1 Penzel T, Pevernagie D, Dogas Z, et al. Catalogue of knowledge and skills for sleep medicine. J Sleep Res 2014; 23: $222-238$. 